Chinese Journal of Organic Chemistry

\title{
甾体肜醚化合物的合成及抗肿瘤活性研究
}

\author{
黄燕敏 $^{a} \quad$ 苏绍烊 ${ }^{b} \quad$ 贾琳怡 ${ }^{c} \quad$ 甘春芳 ${ }^{a}$ \\ 林啟福 ${ }^{a} \quad$ 二斌 ${ }^{a}$ 崔建国*,a \\ ( ${ }^{a}$ 广西师范学院化学与生命科学学院 南宁 530001) \\ $(b$ 广西特种设备监督检验院 南宁 530219) \\ ( ${ }^{c}$ 广西南宁市第三十七中 南宁 530001)
}

\begin{abstract}
摘要 肟醚类化合物往往表现出很好的杀虫、除草、抗菌、抗病毒以及抗肿瘤等生理活性，因而受到人们的广泛关注. 从胆甾醇、豆甾醇出发，通过不同的合成路线，制备得到系列 3-取代、6-取代及 22-取代甲氧基肜醚和苄氧基肟醚甾体 化合物，通过 IR、NMR 及 MS 等现代分析方法对合成物进行了结构表征. 同时，分别采用人胃癌细胞(SGC-7901)、人 肝癌细胞(Bel-7404)和鼻咽癌细胞(CNE-2)对合成产物及部分合成中间体进行了体外抑制肿瘤细胞生长增殖活性研究. 结果表明, 某些具有 22-取代结构的甲氧肜醚及茮氧肜醚甾体化合物对这些肿瘤细胞株表现出较好的抑制活性，其中 22-降-3 $\beta$-差基-22-苠氧肟基-豆甾-6-缩胺硫腙(15)对人鼻咽癌细胞 $\mathrm{CNE}-2$ 的 $\mathrm{IC}_{50}$ 值为 $5.7 \mu \mathrm{mol} / \mathrm{L}$.
\end{abstract}

关键词 胆甾醇; 豆甾醇; 甾体肜醚化合物; 抗肿瘤活性; 生理活性

\section{Synthesis and Antiproliferative Evaluation of Some Steroidal Oxime Ether}

\author{
Huang, Yanmin ${ }^{a} \quad \mathrm{Su}$, Shaoyang $^{b} \quad$ Jia, Linyi $^{c} \quad$ Gan, Chunfang ${ }^{a}$ \\ Lin, Qifu ${ }^{a} \quad$ Kong, Erbin ${ }^{a} \quad$ Cui, Jianguo ${ }^{*, a}$ \\ ( ${ }^{a}$ Chemistry and Life Science College, Guangxi Teachers Education University, Nanning 530001) \\ ( ${ }^{b}$ Guangxi Special Equipment Supervision and Inspection Institute, Nanning 530026) \\ ( ${ }^{c}$ Guangxi Number Thirty-seven Middle School, Naning 530001)
}

\begin{abstract}
Oxime ethers display remarkable insecticidal, weeding, antibacterial, antiviral and anticancer physiological activities. Series of steroidal oxime ethers with the structure of 3-, 6- or 22- $O$-methyloxime or $O$-benzyloxime were designed and synthesized by different synthetic route using cholesterol or stigmasterol as starting materials. Their structures were characterized by IR, NMR and MS. Antiproliferative activities of the compounds were assayed by thiazolyl blue tetrazolium bromide (MTT) method. Some compounds displayed distinct cytotoxicity against SGC-7901, Bel-7404 and CNE-2 cancer cell lines. Our results reveal that the compounds with the structure of 22- $O$-methyloxime and 22-O-benzyloxime have better antiproliferative activities. 3 $\beta$-Hydroxy-22- $O$-benzyloxime-22,23-secostigmastan-6-thiosemicarbazone (15) displays an excellent antiproliferative activity against $\mathrm{CNE}-2$ cancer cells owning an $\mathrm{IC}_{50}$ value of $5.7 \mu \mathrm{mol} / \mathrm{L}$.
\end{abstract}

Keywords cholesterol; stigmasterol; steroidal oxime ether; antiproliferative activity; bioactivity

甾体激素在维持生命、调节性能及免疫功能、对机 体的发育及计划生育等方面有着不可替代的作用，被称 为人类生命的金钥匙. 人们在研究中发现，对甾体的结 构进行不同的改造, 包括在甾核中引入不同的杂原子或 不同官能团，此时甾体化合物本身常常会在生命体中出
现不同的生理活性变化，比如在甾体的不同部位引进肜 类官能团, 会导致化合物出现心脏有着正向肌性作用的 生理活性 ${ }^{[1]}$ 、抗癌活性 $[2,3]$ 、抗菌及避孕等生理活性 ${ }^{[4,5]}$.

肜醚化合物是一类具有显著生理活性的化合物，该 类化合物具有很好的杀虫、杀螨、除草、抗菌、抗病毒

\footnotetext{
*E-mail: cuijg1954@126.com

Received March 22, 2014; revised April 21, 2014; published online May 7, 2014.

Project supported by the Natural Science Foundation of Guangxi Province (No. 2010GXNSFD013019) and the Natural Science Fund of Education Department of Guangxi Province (No. 201202ZD059).

广西自然科学基金(No. 2010GXNSFD013019)和广西教育厅重点基金(No. 201202ZD059)资助项目.
} 
等生物活性 ${ }^{[6]}$, 大多具有高效、低毒、低残留等优点, 因 此肜醚类化合物作为一类农药已经在农业上得到较广 泛的研究和应用. 如美国联邦公司开发生产的肜醚类杀 菌剂霜腿霉(Cymoxan)及除草剂禾草灭(Alloxydim), 它 们都是具有肜醚结构的化合物. 而对于具有肜醚结构的 甾体类肜醚化合物，文献中报道不多. Jindal 等 ${ }^{[7]}$ 合成了 系列具有 3-肜醚及 17-肜醚结构的雄甾烷类衍生物, 发 现该类化合物具有明显的抗肿瘤活性, 对肺癌细胞 (NCI-H23, NCI-H522) 及结肠癌细胞 (SW-620, COLO 205)等多种肿瘤细胞具有显著抑制作用. Lee 等 ${ }^{[8]}$ 合成了 一种具有肜醚结构的甾体激素配合物, 该化合物能成功 透过细胞膜, 并监测基因的转录. Khan 等 ${ }^{[9]}$ 合成了一类 6-肜醚化合物, 此类化合物具有很好的抗菌活性, 其中 当 3-位为氯取代时抗菌效果优于临床用药氯霉素. 周雪 琴等 ${ }^{[10]}$ 合成了一类具有 A-环内酰胺结构的 17-肜醚化合 物, 这些化合物均具有较好的 $5 \alpha$-还原酶抑制活性, 其 中某些化合物的效果与非那雄胺相当. Ivan 等合成了一 类 19-肜醚化合物, 它们具有通过调节受体调节离子通 道的渗透能力 ${ }^{[11]}$, 同时具有调节中枢神经系统的功 能 ${ }^{[12]}$.

我们 ${ }^{[13 \sim 15]}$ 在前期对甾体肟类化合物研究的基础上, 开展了对甾体肜醚类化合物的合成及生理活性研究. 从 天然甾醇豆甾醇及胆甾醇出发, 通过不同的化学反应, 共合成了 18 个分别具有 3-肜醚、6-肜醚或 22-肜醚结构
的甾体肜醚类化合物, 并研究了它们体外抑制肿瘤细胞 生长增殖活性，结果发现某些具有 22-甲基肜基及 22-芐 基肜基的化合物具有很好的体外抑制肿瘤细胞生长增 殖活性.

\section{1 结果与讨论}

\section{1 化学部分}

化合物 $1,8,18$ 及 21 的合成见文献[15].

首先，我们合成了具有 3,22-二甲基肜醚结构的甾 体化合物 3 5 (Scheme 1).

从豆甾-3,6-二酮(1)出发, 通过臭氧化反应使 1 的 22-位双键断裂，生成豆甾-3,6-二酾-22-醛(2). 化合物 2 的结构可从其 NMR 谱得到证实, 在 $\mathbf{2}$ 的 ${ }^{1} \mathrm{H}$ NMR 中, $\delta$ 9.48 处的化学位移是醛基上 $\mathrm{H}$ 的特征峰; 而 ${ }^{13} \mathrm{C} \mathrm{NMR}$ 中 $\delta 204.6$ 的共振吸收进一步证实了 22-醛基的生成. 2 进一步与甲氧氨盐酸盐反应, 得到具有 3,22-二甲氧豚 醚结构的化合物 3.3 的结构从 ${ }^{1} \mathrm{H}$ NMR 中化学位移为 $\delta$ 3.82 和 3.84 的两个与氧相连的甲基单峰得到证实. 另 外，从化合物 3 的 ${ }^{1} \mathrm{H}$ NMR 和 ${ }^{13} \mathrm{C}$ NMR 中都没有发现异 构体的存在. 从化合物 $\mathbf{2}$ 的结构来看, 22-醛基比 3-羰基 及 6-羰基更为活泼, 易于发生反应. 而从化合物 2 中 3羊基和 6-羰基的空间位阻来看, 6-羰基相邻的 5-位碳是 一个叔碳，同时由于 19-甲基及 5-位及 7-位 $a$-键氢的影<smiles>CCC(/C=C/[C@@H](C)C1CCC2C3CC(=O)C4CC(=O)CCC4(C)C3CCC21C)C(C)C</smiles><smiles>CC(C=O)C12CCC3C(CC(=O)C4CC(=O)CCC43C)C1CCC2C</smiles><smiles>CN=C1CC[C@@]2(C)C(C1)C(=O)CC1C3CC[C@H](C(C)/C=N/OC)C3(C)CCC12</smiles>

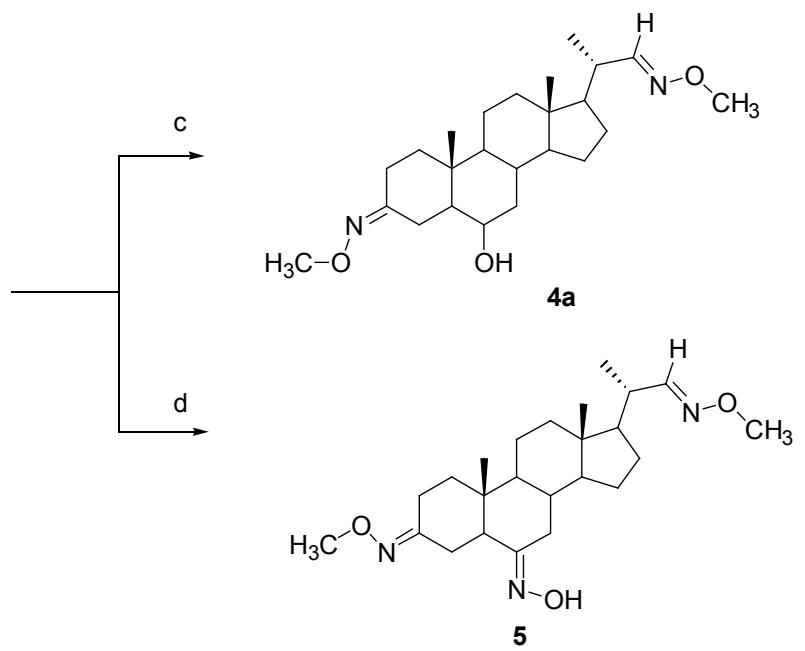<smiles>CCC1C2CC(O)C3C/C(=N/OC)CCC3C2CC[C@@H]1[C@@H](C)/C=N/OC</smiles>

4b

Reagents and conditions: (a) 1) $\mathrm{O}_{3} /-78{ }^{\circ} \mathrm{C}, \mathrm{CH}_{2} \mathrm{Cl}_{2} / \mathrm{CH}_{3} \mathrm{OH}\left(V: V=4\right.$ : 1); 2) $\mathrm{Me}_{2} \mathrm{~S}$; (b) $\mathrm{NH}_{2} \mathrm{OCH}_{3} \cdot \mathrm{HCl} / 95 \%$ EtOH/NaOAc$\cdot 3 \mathrm{H}_{2} \mathrm{O}$; (c) $\mathrm{NaBH}_{4}, \mathrm{CH}_{3} \mathrm{OH}$; (d) $\mathrm{NH}_{2} \mathrm{OH} \cdot \mathrm{HCl} / 95 \% \mathrm{EtOH} / \mathrm{NaOAc} \cdot 3 \mathrm{H}_{2} \mathrm{O}$

Scheme 1 
响, 因此空间位阻较大. 而相对于 3-羰基来看, 其相邻 的 2-位碳及 4-位碳均是仲碳, 同时 2,4-位上的 $a$-键氢离 3 -羰基较远, 因此空间位阻较小, 所以反应主要发生在 3-位羰基(图 1).

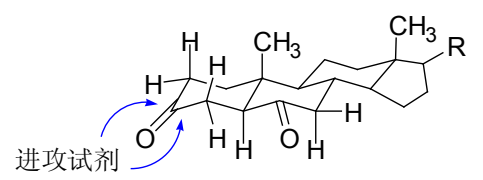

图 1 化合物 $\mathbf{2}$ 的构象式

Figure 1 The conformation of compound 2

22-降-6-氧代豆甾-3,22-甲氧基肜(3)通过硼氢化钠 还原，得到化合物 4 . 有趣的是，从化合物 4 中分离得到 异构体 $4 \mathrm{a}$ 和 $4 \mathrm{~b}$, 化合物 $4 \mathrm{a}$ 和 $4 \mathrm{~b}$ 中两个甲氧肜基保留, 仅仅是 6-羰基被还原为羟基. 从 $4 \mathrm{a}$ 和 $4 \mathrm{~b}$ 的 ${ }^{13} \mathrm{C} \mathrm{NMR}$ 中 明显可以看到, 3 在 $\delta 209.9$ 的 6-羰基化学位移已经消失, 代之为 $\delta 71.0$ 和 70.9 的 6-羟基碳的化学位移. 从 ${ }^{1} \mathrm{H}$ NMR 中 $\delta 3.80 \sim 3.83$ 与羟基相连碳上氢的化学位移, 进 一步证实化合物 3 的 6-羰基已经被还原成为 6-羟基，而 $\delta 3.79$ 和 3.81 两个与氧相连甲基单峰的存在, 表明两个 甲氧肜基在反应中没有被还原.

从 $4 \mathbf{a}^{1} \mathrm{H}$ NMR 中 $\delta 2.93(\mathrm{dd}, J=15.0,3.3 \mathrm{~Hz}, 1 \mathrm{H})$ 的 化学位移分析, 可认定为 $\mathrm{C}_{4}-\beta-\mathrm{H}$ 的共振吸收峰, 其在 $\mathrm{C}_{4}-\alpha-\mathrm{H}$ 和 $\mathrm{C}_{5}-\mathrm{H}$ 的作用下形成了 $\mathrm{dd}$ 峰, 同时由于 $3-\mathrm{OCH}_{3}$ 的去屏蔽影响移向低场, 其构型为 $(3 Z, 22 E)$-22-降-6-差圣 基豆甾-3,22-二甲氧基肟. 同样分析，化合物 4b 构型为 (3E,22E)-22-降-6-羊基豆甾-3,22-二甲氧基肜. 化合物 3 与盐酸差胺反应，则得到化合物 5 , 反应中 3 的 6 -羰基 被转变为 6-肜基, 其结构从 NMR 谱分析, 5 具有 $(3 E, 6 E, 22 E)$-构型，可能是此种构型可以使 3-甲氧肜基 中的甲氧基和 6-肜基中的羟基达到最大程度的远离, 使
产物更加稳定. 反应中未有分离得到另一(3Z)-异构体.

从化合物 2 出发，在三水乙酸钠存在下与茮氧胺盐 酸盐反应，得到化合物 6 . 从 6 的 NMR 谱中可以明显看 到, 6 是一个由 $(3 E, 22 E)$-及 $(3 Z, 22 E)$-异构体构成的混合 物，两者的比例大约为 $1: 1$. 化合物 6 没有经过分离, 直接通过 $\mathrm{NaBH}_{4}$ 还原，得到 6-羰基被还原的产物 7, 产 物 7 进一步分离得到异构体 7a 和 7b (Scheme 2). 在 7a 的 ${ }^{1} \mathrm{H}$ NMR 谱中, 在 $\delta 3.26(J=15.3,3.0 \mathrm{~Hz}, 1 \mathrm{H})$ 处出现 一个 $\mathrm{dt}$ 吸收峰, 这可认定为 $\mathrm{C}_{2}-\beta-\mathrm{H}$ 的化学位移, $7 \mathbf{a}$ 具有 (3E)-的苠氧肟基结构. 而在 $7 \mathbf{b}$ 的 ${ }^{1} \mathrm{H} \mathrm{NMR}$ 谱中, 在 $\delta$ $3.05(J=15.0,3.0 \mathrm{~Hz}, 1 \mathrm{H})$ 处存在一个 $\mathrm{dd}$ 峰, 可认定为 $\mathrm{C}_{4}-\beta-\mathrm{H}$ 的化学位移, 即 $7 \mathbf{b}$ 具有 $(3 Z)$-构型.

另外，从化合物 $\mathbf{8}$ 出发通过 3-差基乙酰化保护得到 化合物 9, 9 经过臭氧化反应得到 22-双键断裂的产物 $\mathbf{1 0}$, 10 的结构从其 ${ }^{1} \mathrm{H}$ NMR 中 $\delta 9.60(\mathrm{~d}, J=3.0 \mathrm{~Hz}, 1 \mathrm{H})$ 的低 场化学位移得到证实, 这是 22-双键发生断裂后形成醛 基的标志. 从 10 的 ${ }^{13} \mathrm{C} \mathrm{NMR}$ 来看, $\delta 204.7$ 是 22-醛基中 羰基碳的化学位移, 而 $\delta 210.0$ 是 6-位羰基碳的化学位 移. 化合物 10 与等物质的量的茮氧基胺反应得到化合 物 11, 从 11 的 ${ }^{1} \mathrm{H}$ NMR 来看, $\delta 5.04$ 处 2 个氢的积分面 积是苠基中 $\mathrm{CH}_{2}$ 的化学位移, 而低场 $\delta 7.35 \sim 7.29$ 中 5 个氢积分面积是苯环上 $\mathrm{H}$ 的化学位移. 另外, 从其 ${ }^{13} \mathrm{C}$ $\mathrm{NMR}$ 来看, 我们清楚地看到, 在化合物 10 中 $\delta 204.7$ 醛 羰基碳的化学位移已经消失, 在 $\delta 155.8$ 出现了新的 22碳的信号, 说明 22-醛基已经转变成为肟醚. 而 $\delta 210.1$ 的 6-酮羰基碳化学位移的保留, 进一步说明在这里是 22-醛基发生了反应，而6-酮羰基没有发生变化. 对化合 物 11 的 6-位羰基进行不同的官能团转换，可分别得到 具有 6-肜基结构的化合物 12, 具有 6-氨基硫腙结构的化 合物 14 和具有 6-甲氧肜醚结构的化合物 16. 进一步对
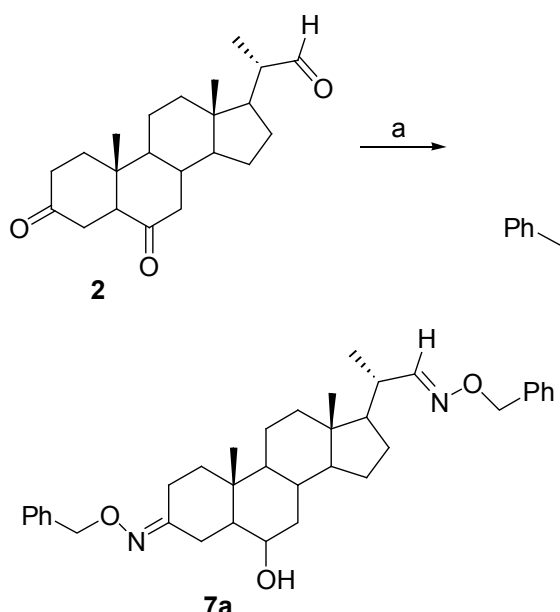<smiles>C[C@H](/C=N/OCc1ccccc1)C1CCC2C3CC(=O)C4C/C(=N/OCc5ccccc5)CC[C@]4(C)C3CC[C@@]21C</smiles>
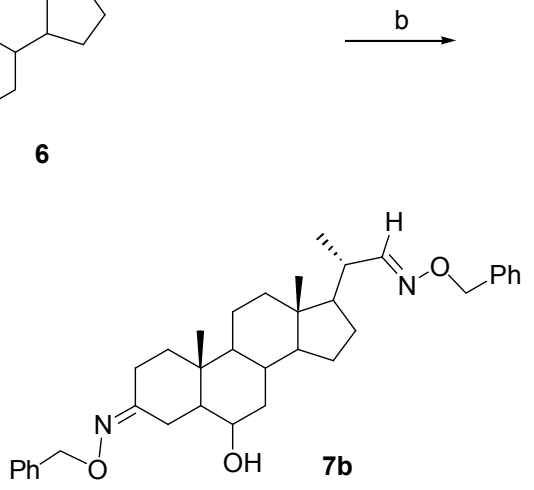

Reagents and conditions: (a) $\mathrm{NH}_{2} \mathrm{OCH}_{2} \mathrm{C}_{6} \mathrm{H}_{5} \cdot \mathrm{HCl} / 95 \% \mathrm{EtOH} / \mathrm{NaOAc} \cdot 3 \mathrm{H}_{2} \mathrm{O}$; (b) $\mathrm{NaBH}_{4}, \mathrm{CH}_{3} \mathrm{OH}$

Scheme 2 
<smiles>CCC(C)C(C)(C)C=CC1CCC2C3CC(=O)C4CC(O)CCC4(C)C3CCC12C</smiles>

8

10

11

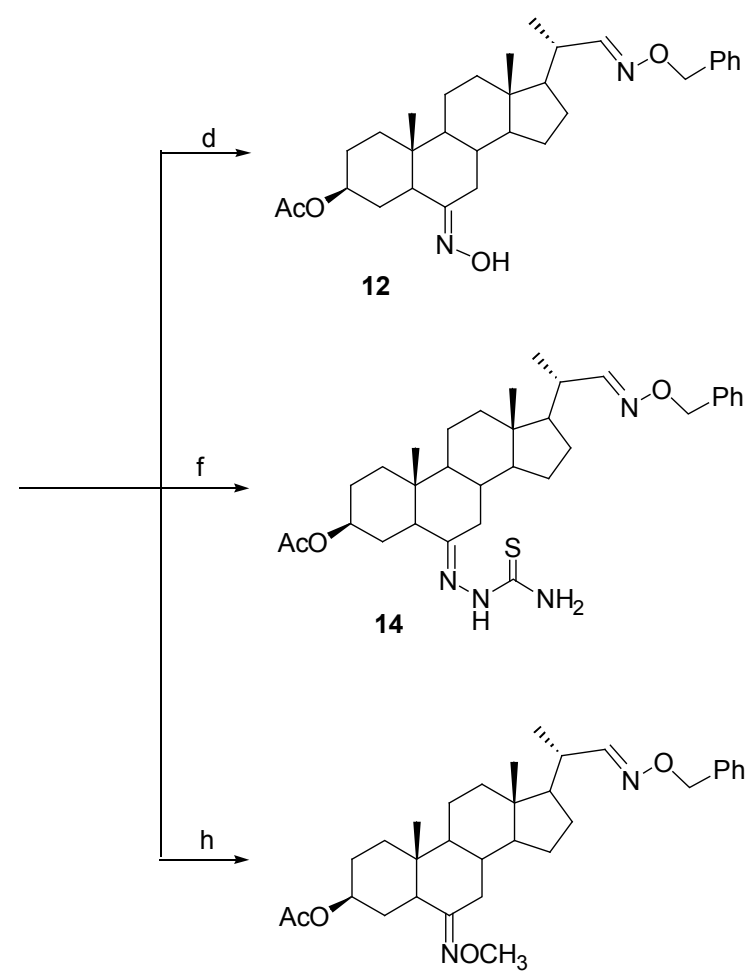

16

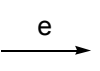<smiles>C[C@H](/C=N/OCc1ccccc1)C1CCC2C3C/C(=N\O)C4C[C@@H](O)CC[C@]4(C)C3CCC21C</smiles>

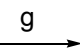<smiles>C[C@H](/C=N/OCc1ccccc1)C1CCC2C3C/C(=N/NC(N)=S)C4C[C@@H](O)CC[C@]4(C)C3CCC21C</smiles>

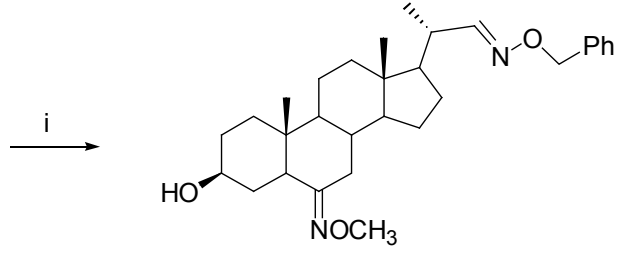

17

Reagents and conditions: (a) $\mathrm{Ac}_{2} \mathrm{O} / \mathrm{Py}$; (b) 1) $\mathrm{O}_{3} /-78{ }^{\circ} \mathrm{C}, \mathrm{CH}_{2} \mathrm{Cl}_{2} / \mathrm{CH}_{3} \mathrm{OH}\left(V: V=4: 1\right.$ ); 2) $\mathrm{Me}_{2} \mathrm{~S}$; (c) $\mathrm{NH}_{2} \mathrm{OCH}_{2} \mathrm{C}_{6} \mathrm{H}_{5} \cdot \mathrm{HCl}_{2} / 95 \% \mathrm{EtOH} /$ $\mathrm{NaOAc} \cdot 3 \mathrm{H}_{2} \mathrm{O}$; (d) $\mathrm{NHOH} \cdot \mathrm{HCl} / 95 \% \mathrm{EtOH} / \mathrm{NaOAc} \cdot 3 \mathrm{H}_{2} \mathrm{O}$; (e) $\mathrm{KOH} / \mathrm{CH}_{3} \mathrm{OH}\left(V: V=5: 95\right.$ ); (f) $\mathrm{NH}_{2} \mathrm{NHCSNH}_{2} / \mathrm{C}_{2} \mathrm{H}_{5} \mathrm{OH} / \mathrm{CH} 3 \mathrm{COOH}, \mathrm{pH}=4$; (g) $\mathrm{KOH} / \mathrm{CH}_{3} \mathrm{OH}(V: V=5: 95)$; (h) $\mathrm{NH}_{2} \mathrm{OCH}_{3} \cdot \mathrm{HCl} / 95 \% \mathrm{EtOH} / \mathrm{NaOAc} \cdot 3 \mathrm{H}_{2} \mathrm{O}$; (i) $\mathrm{KOH} / \mathrm{CH}_{3} \mathrm{OH}(V: V=5: 95)$

\section{Scheme 3}

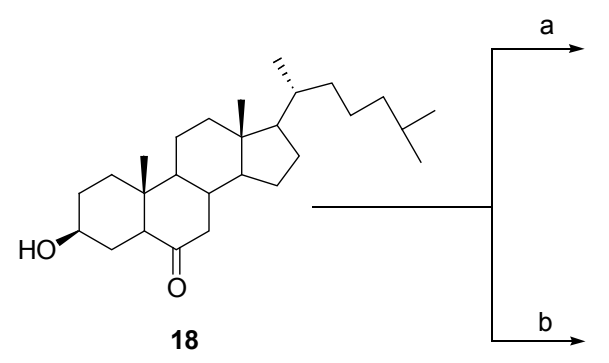<smiles>CON=C1CC2C(CCC3(C)C(CCCC(C)C)CCC23)C(C)C1CCC(C)C</smiles><smiles>CC(C)CCC[C@H](C)C1CCC2C3C/C(=N\OCc4ccccc4)C4C[C@@H](O)CC[C@]4(C)C3CCC21C</smiles>

Reagents and conditions: (a) $\mathrm{CH}_{3} \mathrm{ONH}_{2} \cdot \mathrm{HCl} / 95 \% \mathrm{EtOH}, \mathrm{NaOAc}, 65{ }^{\circ} \mathrm{C}$; (b) $\mathrm{ArCH}_{2} \mathrm{ONH}_{2} \cdot \mathrm{HCl} / 95 \% \mathrm{EtOH}, \mathrm{NaOAc}, 65{ }^{\circ} \mathrm{C}$

Scheme 4 


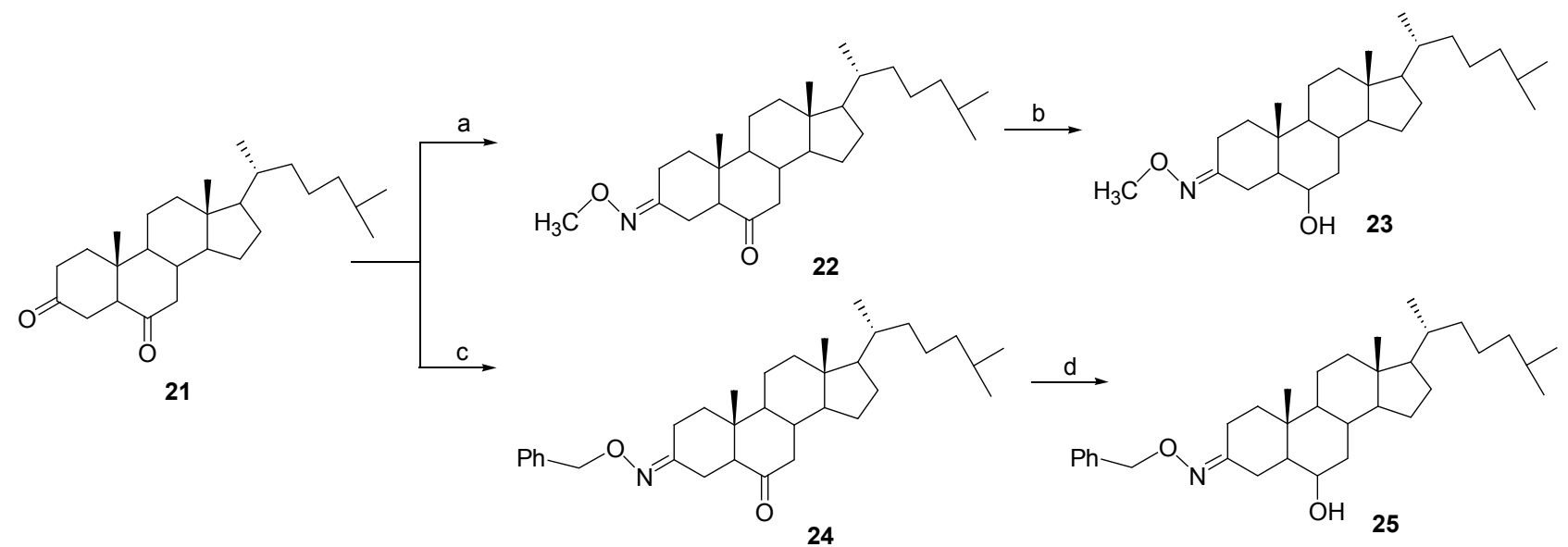

Reagents and conditions: (a) $\mathrm{CH}_{3} \mathrm{ONH}_{2} \cdot \mathrm{HCl} / 95 \% \mathrm{EtOH}, \mathrm{NaOAc} 65{ }^{\circ} \mathrm{C}$; (b) $\mathrm{NaBH}_{4}, \mathrm{CH}_{3} \mathrm{OH}$; (c) $\mathrm{ArCH}_{2} \mathrm{ONH}_{2} \cdot \mathrm{HCl} / 95 \% \mathrm{EtOH}, \mathrm{NaOAc}, 65{ }^{\circ} \mathrm{C}$; (d) $\mathrm{NaBH}$, $\mathrm{CH}_{3} \mathrm{OH}$

\section{Scheme 5}

12,14 和 16 的 3-位乙酰氧基水解去保护分别得到化合 物 13, 15 和 17. 化合物 12 17 的结构都从它们的光谱、 波谱及质谱数据得到表征确定.

为了探索不同取代位置结构的肜醚取代基对甾体 化合物抑制肿瘤细胞生长增殖活性的影响. 从化合物 18 出发, 化合物 18 分别与甲氧胺盐酸盐及芐氧胺盐酸 盐反应, 得到化合物 19 和 $\mathbf{2 0}$. 从 ${ }^{1} \mathrm{H}$ NMR 谱来看, 化合 物 19 在 $\delta 3.21(J=13.8,4.5 \mathrm{~Hz}, 1 \mathrm{H})$ 处及化合物 20 在 $\delta$ $3.29(J=13.3,4.5 \mathrm{~Hz}, 1 \mathrm{H})$ 处存在的 $\mathrm{dd}$ 峰化学位移可推 断为 $\mathrm{C}_{7}-\alpha-\mathrm{H}$ 的位移信号, 它们在肟醚键中 $\mathrm{O}$ 原子的负 诱导效应影响下移向低场, 这说明化合物 19 和 20 具有 6- $E$ 构型.

从化合物 21 出发, 分别与甲氧胺盐酸盐和芐氧胺 盐酸盐反应，控制适量的反应的物质的量比，分别得到 化合物 22 和 24, 22 和 24 进一步通过嗍氢化钠还原, 得 到 6-羰基还原产物 23 和 $\mathbf{2 5}$, 反应过程中 3-肜醚结构保 留. 化合物 $21 \sim 25$ 的结构都通过光谱、波谱及质谱数据 得到表征.

\section{2 生物部分}

分别采用人胃癌细胞(SGC-7901)、肝癌细胞 (Bel-7404)和鼻咽癌细胞(CNE-2), 使用 MTT 法对所合 成的目标产物及部分中间体进行体外抑制肿瘤细胞生 长增殖活性测试, 结果如表 1 所示. 从表中数据可以发 现：对于拥有 3,22-二甲氧肜醚结构的化合物 3 5 来说, 具有 6-着基取代的化合物 4a 和 4b 对 SGC-7901 和 Bel-7404 细胞具有明显的抑制生长增殖活性, 但是对鼻 咽癌细胞 CNE-2 则抑制活性不明显. 具有 6-肜基取代的 化合物 5 对此三种细胞株都具有较好的抑制作用，而具 有 6-羰基取代的化合物 3 却没有明显的抑制活性. 原因 可能是羰基还原为羟基及转换为肜基后，一方面加大了
化合物的水溶度，另一方面由于羟基和肜基易于与细胞 中的蛋白质或 DNA 形成分子间氢键，会增强化合物与 蛋白质或 DNA 的亲合作用，进一步增加化合物的细胞 毒性. 从具有 3,22-二茮氧肜醚结构的立体异构体 $\mathbf{7 a}$ 和 $7 \mathbf{b}$ 来看, 具有 $3 Z$ 构型的化合物 $7 \mathbf{b}$ 比具有 $3 E$ 构型的异 构体 7a 对 SGC-7901 和 CNE-2 细胞具有更好的抑制活 性. 对 Bel-7404 细胞来说, 6-㷇基还原后的产物 $7 \mathbf{a}$ 和 7b 比它们还原的前体化合物 6 的抑制活性要好, 这进一步 说明羟基的存在对提高化合物细胞毒性的重要性.

对于 22-芐氧肜醚类化合物来说，具有 6-肜基取代 的化合物 12 及具有 6 -氨基硫腙基取代的化合物 15 具有 较好的细胞毒性, 特别是化合物 15 对鼻咽癌细胞 CNE-2 抑制生长增殖作用的 $\mathrm{IC}_{50}$ 值为 $5.7 \mu \mathrm{mol} / \mathrm{L}$, 优于 阳性对照物顺铂，具有一定的抑制选择性，对此在进一 步研究探讨. 化合物 13 和化合物 $\mathbf{1 5}$ 相比较, 明显地化 合物 15 对 Bel-7404 及 CNE-2 细胞的抑制活性要高于化 合物 13, 说明 6-氨基硫腙基取代比 6-肜基取代具有更强 的细胞毒性，是更好的活性基团.

另外，对于具有胆甾烷支链结构的甾体化合物来 说，具有 6-肜醚取代结构的化合物 19 和 20 对胃癌细胞 SGC-7901 表现出一定的抑制活性. 然而，同样具有 3,6二取代结构的特征，取代官能团相同但是取代位置刚好 相反的 3-肜醚类甾体化合物 $\mathbf{2 3}$ 和 $\mathbf{2 5}$ 对此种细胞却没有 表现出明显的抑制作用. 由此可见对于甾体化合物来 说，甾核中官能团取代位置的不同，对化合物的生理活 性会产生较大的影响.

从表 1 结果还可以看到, 具有 22-肜醚取代结构的 此类甾体化合物比具有胆甾烷支链结构的此类肜醚化 合物具有更好的细胞毒性，这可能是前者比后者具有更 大水溶性的原因, 还需要我们进一步的研究探讨. 
表 1 目标产物的体外抗肿瘤活性 $\left[\mathrm{IC}_{50} /\left(\mu \mathrm{mol} \cdot \mathrm{L}^{-1}\right)\right]^{a}$

Table 1 In vitro antiproliferative activities $\left[\mathrm{IC}_{50} /\left(\mu \mathrm{mol} \cdot \mathrm{L}^{-1}\right)\right]$ of target compounds

\begin{tabular}{cccl}
\hline Compound & SGC-7901 & Bel-7404 & \multicolumn{1}{c}{ CNE-2 } \\
\hline $\mathbf{3}$ & $>100$ & $>100$ & $>100$ \\
$\mathbf{4 a}$ & 17.3 & 24.7 & $>100$ \\
$\mathbf{4 b}$ & 69.1 & 17.3 & $>100$ \\
$\mathbf{5}$ & 19 & 14 & 14.1 \\
$\mathbf{6}$ & 44.9 & $>100$ & $>100$ \\
$\mathbf{7 a}$ & $>100$ & 18.4 & $>100$ \\
$\mathbf{7 b}$ & 40.5 & 29.5 & 84.7 \\
$\mathbf{1 1}$ & 30.4 & 22.3 & $>100$ \\
$\mathbf{1 2}$ & 15.2 & 13.3 & 76.2 \\
$\mathbf{1 3}$ & 14.5 & $>100$ & 99.6 \\
$\mathbf{1 4}$ & 68.8 & $>100$ & 74.1 \\
$\mathbf{1 5}$ & 21.0 & 45.6 & 5.7 \\
$\mathbf{1 6}$ & 26.8 & $>100$ & $>100$ \\
$\mathbf{1 7}$ & 27.1 & $>100$ & $>100$ \\
$\mathbf{1 9}$ & 48.6 & $>100$ & $\mathrm{ND}$ \\
$\mathbf{2 0}$ & 52 & 38 & $\mathrm{ND}$ \\
$\mathbf{2 3}$ & $>100$ & 72.0 & $\mathrm{ND}$ \\
$\mathbf{2 5}$ & $>100$ & 80 & $\mathrm{ND}$ \\
Cisplatin & 6.7 & 10.1 & 16.8 \\
\hline${ }^{a}$ Data represent the mean values of three independent determinations; ${ }^{b}$ Not \\
determined. & & &
\end{tabular}

\section{2 结论}

采用不同的合成路线, 我们分别合成了某些具有 3取代、6-取代及 22-取代甲氧基肜醚和芐氧基肜醚结构 的甾体化合物, 这些化合物的结构通过现代分析方法得 到了表征确认. 同时, 对合成产物及部分合成中间体进 行体外抑制肿瘤细胞生长增殖活性研究表明, 某些具有 22-取代结构的甲氧肜醚及苄氧肜醚甾体化合物对人胃 癌细胞(SGC-7901)、肝癌细胞(Bel-7404)和鼻咽癌细胞 (CNE-2)具有较好的抑制生长增殖活性; 具有 22-肜醚取 代结构的化合物比具有胆甾烷支链结构的肜醚化合物 具有更好的细胞毒性. 研究结果为我们设计合成具有更 好抗肿瘤活性的甾体化合物提供了有用的参考.

\section{3 实验部分}

\section{1 化学合成部分}

\subsection{1 试剂和仪器}

合成所用试剂如无特别说明均为分析纯, 溶剂按常 规方法进行处理干燥. IR 测定采用 Nicolet FT-360 傅立 叶红外光谱仪; 熔点测定采用 $\mathrm{X}_{6}$ 显微熔点测定仪, 温度 计未经校正; ${ }^{1} \mathrm{H}$ NMR, ${ }^{13} \mathrm{C}$ NMR 测定采用德国 Bruker AV-500 型和 Bruker AV-300 型超导核磁共振仪, 化学位 移值用 $\delta$ 表示, 偶合常数 $J$ 用 $\mathrm{Hz}$ 表示; 高分辨质谱采用 美国 Agilent 6210 TOFMS 质谱仪.

化合物 $1,8,18,21$ 的合成见参考文献[15].
3.1.2 22-降-3,6-二氧代豆甾-22-醛(2)的合成

将 $500 \mathrm{mg}(1.17 \mathrm{mmol})$ 化合物 $\mathbf{1}$ 加入带有支管的圆 底烧瓶中, 加入 $16 \mathrm{~mL}$ 二氯甲烷和 $4 \mathrm{~mL}$ 甲醇, 溶解完全 后置于液氮冷却的乙酸乙酯保温瓶中, 待体系温度冷却 到 $-78{ }^{\circ} \mathrm{C}$ 后，通入富含有 $\mathrm{O}_{3}$ 的氧气流，TLC 跟踪反应 $[V($ 石油醚 $): V($ 乙酸乙酯 $)=4: 1]$, 溶液变为淡蓝色后 停止通入 $\mathrm{O}_{3}$, 继续通入氧气 $20 \mathrm{~min}$, 待体系升至室温, 加入 $3 \mathrm{~mL}$ 二甲硫醚, 搅拌过夜. 减压蒸去大部分溶剂, 得到白色固体的混合油状物, 该油状物用 $60 \mathrm{~mL}$ 二氯甲 烷溶解, 并依次用水、饱和氯化钠水溶液洗, 有机层用 无水硫酸钠干燥. 减压蒸去溶剂, 得到无色透明油状物. 快速柱层析分离 [洗脱剂: $V$ (石油醚) $: V($ 乙酸乙酯 $)=$ 4: 1]得到固体化合物 $2242 \mathrm{mg}$, 产率 60\%. m.p. 168 $169{ }^{\circ} \mathrm{C} ;{ }^{1} \mathrm{H}$ NMR (DMSO- $\left.d_{6}, 500 \mathrm{MHz}\right) \delta: 0.69$ (s, $3 \mathrm{H}$, $\left.18-\mathrm{CH}_{3}\right), 0.87\left(\mathrm{~s}, 3 \mathrm{H}, 19-\mathrm{CH}_{3}\right), 1.05(\mathrm{~d}, J=6.5 \mathrm{~Hz}, 3 \mathrm{H}$, $\left.21-\mathrm{CH}_{3}\right), 2.78\left(\mathrm{dd}, J=13.5,4.0 \mathrm{~Hz}, 1 \mathrm{H}, \mathrm{C}^{5}-\mathrm{H}\right), 9.54(\mathrm{~d}$, $\left.J=6.5 \mathrm{~Hz}, 1 \mathrm{H}, \mathrm{C}^{22}-\mathrm{H}\right) ;{ }^{13} \mathrm{C}$ NMR $\left(\mathrm{CDCl}_{3}, 125 \mathrm{MHz}\right) \delta$ : 209.4 (3-C), 209.3 (6-C), 205.7 (22-C), 56.5 (14-C), 55.5 (5-C), 52.4 (17-C), 51.0 (20-C), 49.0 (13-C), 46.2 (9-C), 43.5 (7-C), 41.0 (10-C), 39.0 (4-C), 37.7 (12-C), 37.6 (2-C), 37.4 (1-C), 37.2 (8-C), 26.9 (16-C), 24.4 (15-C), 21.6 (11-C), 13.6 (19-C), 12.6 (18-C), 12.5 (21-C); IR (KBr) $v$ : 2931, 2871, 2734, 1709, 1463, 1386, 1265, 1227, $920 \mathrm{~cm}^{-1}$.

\subsubsection{2-降-6-氧代豆甾-3,22-甲氧基肪(3)的合成}

将 $100 \mathrm{mg}(0.29 \mathrm{mmol})$ 化合物 2 溶于 $20 \mathrm{~mL} 95 \%$ 乙 醇, 然后加入三水乙酸钠 $79 \mathrm{mg}(0.58 \mathrm{mmol})$, 加热搅拌 溶解, 待溶解后分批缓慢加入甲氧基胺盐酸盐 $51 \mathrm{mg}$ (0.58 mmol), 控制反应温度在 $65{ }^{\circ} \mathrm{C}$ 左右, TLC 跟踪反 应, $1 \mathrm{~h}$ 后停止反应. 减压抽除大部分乙醇, 出现大量白 色固体, 加入少许蒸馏水, 有白色沉淀生成, 用乙酸乙 酯萃取 $(20 \mathrm{~mL} \times 3)$, 合并有机层, 有机层依次用水洗, 饱和食盐水各洗一次, 然后用无水硫酸钠干燥. 过滤, 减压蒸去溶剂, 得到白色固体. 将此固体采用柱层析分 离[洗脱剂: $V$ (石油醚) $: V$ (乙酸乙酯 $)=8: 1$ ], 得到化合 物 $3200 \mathrm{mg}$, 产率 67.1\%. m.p. $120 \sim 122{ }^{\circ} \mathrm{C}$; ${ }^{1} \mathrm{H}$ NMR $\left(300 \mathrm{MHz}, \mathrm{CDCl}_{3}\right) \delta: 0.73\left(\mathrm{~s}, 3 \mathrm{H}, 18-\mathrm{CH}_{3}\right), 0.85(\mathrm{~s}, 3 \mathrm{H}$, $\left.19-\mathrm{CH}_{3}\right), 1.05\left(\mathrm{~d}, J=6.6 \mathrm{~Hz}, 3 \mathrm{H}, 21-\mathrm{CH}_{3}\right), 2.37 \sim 2.30(\mathrm{~m}$, $3 \mathrm{H}, \mathrm{C}^{5}-\mathrm{H}$ 及 $\left.\mathrm{C}^{7}-\mathrm{H}\right), 3.22 \sim 3.10\left(\mathrm{~m}, 2 \mathrm{H}, \mathrm{C}^{2}-\beta-\mathrm{H}\right.$ 及 $\left.\mathrm{C}^{4}-\beta-\mathrm{H}\right)$, $3.82\left(\mathrm{~s}, 3 \mathrm{H}, 22-=\mathrm{NOCH}_{3}\right), 3.84\left(\mathrm{~s}, 3 \mathrm{H}, 3-=\mathrm{NOCH}_{3}\right), 6.43$ $\left(\mathrm{d}, J=8.4 \mathrm{~Hz}, 1 \mathrm{H}, \mathrm{C}^{22}-\mathrm{H}\right) ;{ }^{13} \mathrm{C}$ NMR $\left(75 \mathrm{MHz}, \mathrm{CDCl}_{3}\right) \delta$ : 209.9 (6-C), 158.3 (3-C), 155.7 (22-C), 61.5 (22-=NO$\left.\mathrm{CH}_{3}\right), 61.1\left(3-=\mathrm{NOCH}_{3}\right), 58.0(5-\mathrm{C}), 56.5(14-\mathrm{C}), 53.9$ (17-C), 53.6 (13-C), 46.6 (9-C), 43.1 (7-C), 41.8 (12-C), 39.2 (10-C), 37.8 (8-C), 37.1 (20-C), 32.2 (3-C), 26.8 
(4-C), 26.8 (2-C), 24.1 (1-C), 21.4 (16-C), 20.4 (15-C), 17.2 (21-C), 12.4 (19-C), 12.3 (18-C); IR (KBr) v: 3407, 2933, 2864, 1711, 1634, 1458, 1433, 1380, 1303, 1241, $1041 \mathrm{~cm}^{-1}$; HREIMS calcd for $\mathrm{C}_{24} \mathrm{H}_{39} \mathrm{~N}_{2} \mathrm{O}_{3}[\mathrm{M}+\mathrm{H}]^{+}$ 403.2961, found 403.2959.

\subsubsection{2-降-6-羟基豆留-3,22-二甲氧基肪(4)的合成}

室温下将 $200 \mathrm{mg}(0.40 \mathrm{mmol})$ 化合物 3 溶解于 20 $\mathrm{mL}$ 无水甲醇中(其中加入约 $2 \mathrm{~mL}$ 二氯甲烷助溶), 待溶 解完全后, $20 \mathrm{~min}$ 内分批加入 $31 \mathrm{mg}(0.80 \mathrm{mmol}) \mathrm{NaBH}_{4}$. $\mathrm{TLC}$ 监测反应，无原料点随即用 $1 \mathrm{~mol} / \mathrm{L} \mathrm{HCl}$ 淬灭反应， 减压蒸去大部分甲醇, 用乙酸乙酯萃取 $(20 \mathrm{~mL} \times 3)$, 合 并有机层, 有机层用蒸馏水、饱和食盐水各洗一次, 无 水硫酸钠干燥. 减压蒸去溶剂, 得到白色固体, 将此固 体采用柱层析分离[洗脱剂: $V$ (石油醚) $: V$ (乙酸乙酯 $)=$ $6: 1$ ]得到化合物(3Z,22E)-22-降-6-羟基豆甾-3,22-二甲 氧基肜(4a) $68 \mathrm{mg}$, 产率 34.2\%. m.p. $149 \sim 150{ }^{\circ} \mathrm{C} ;{ }^{1} \mathrm{H}$ NMR $\left(300 \mathrm{MHz}, \mathrm{CDCl}_{3}\right) \delta: 0.74\left(\mathrm{~s}, 3 \mathrm{H}, 18-\mathrm{CH}_{3}\right), 1.09(\mathrm{~s}$, $\left.3 \mathrm{H}, 19-\mathrm{CH}_{3}\right), 1.11\left(\mathrm{~d}, J=8.1 \mathrm{~Hz}, 3 \mathrm{H}, 21-\mathrm{CH}_{3}\right), 2.36(\mathrm{~m}$, $\left.1 \mathrm{H}, \mathrm{C}^{20}-\mathrm{H}\right), 2.93\left(\mathrm{dd}, J=15.0,3.3 \mathrm{~Hz}, 1 \mathrm{H}, \mathrm{C}^{4}-\beta-\mathrm{H}\right), 3.79$ (s, $3 \mathrm{H}, 3-\mathrm{NOCH}_{3}$ ), 3.81 (s, $3 \mathrm{H}, 22-\mathrm{NOCH}_{3}$ ), 3.80 3.84 $\left(\mathrm{m}, 1 \mathrm{H}, \mathrm{C}^{6}-\mathrm{H}\right), 7.18\left(\mathrm{~d}, J=8.4 \mathrm{~Hz}, 1 \mathrm{H}, \mathrm{C}^{22}-\mathrm{H}\right) ;{ }^{13} \mathrm{C} \mathrm{NMR}$ $\delta: 160.2$ (3-C), 155.5 (22-C), 71.0 (6-C), 61.1 (22-NO$\left.\mathrm{CH}_{3}\right), 61.0$ (3- $\left.\mathrm{NOCH}_{3}\right), 55.9$ (14-C), 53.9 (17-C), 47.8 (9-C), 42.9 (5-C), 42.8 (13-C), 39.8 (7-C), 39.7 (10-C), 39.6 (12-C), 37.1 (8-C), 36.1 (20-C), 30.2 (4-C), 27.8 (2-C), 27.6 (1-C), 25.2 (16-C), 24.3 (15-C), 20.9 (11-C), 18.1 (21-C), 14.9 (19-C), 12.4 (18-C); IR (KBr) v: 3440, 2933, 2868, 2806, 1638,1560, 1417, 1045, $886 \mathrm{~cm}^{-1}$; HREIMS calcd for $\mathrm{C}_{24} \mathrm{H}_{41} \mathrm{~N}_{2} \mathrm{O}_{3}[\mathrm{M}+\mathrm{H}]^{+}$405.3117, found 405.3106 .

同时, 分离得到异构体(3E,22E)-22-降-6-羊基豆甾3,22-二甲氧基肪(4b) $58 \mathrm{mg}$, 产率 29.1\%. m.p. 168 170 ${ }^{\circ} \mathrm{C} ;{ }^{1} \mathrm{H}$ NMR $\left(300 \mathrm{MHz}, \mathrm{CDCl}_{3}\right) \delta: 0.74$ (s, $\left.3 \mathrm{H}, 18-\mathrm{CH}_{3}\right)$, $1.10\left(\mathrm{~s}, 3 \mathrm{H}, 19-\mathrm{CH}_{3}\right), 1.11$ (d, 3H, $\left.J=6.6,21-\mathrm{CH}_{3}\right), 2.36$ $\left(\mathrm{m}, 1 \mathrm{H}, \mathrm{C}^{20}-\mathrm{H}\right), 2.55\left(\mathrm{t}, 1 \mathrm{H}, J=14.1 \mathrm{~Hz}, \mathrm{C}^{4}-\alpha-\mathrm{H}\right), 3.16 \sim$ $3.10\left(\mathrm{~m}, 1 \mathrm{H}, \mathrm{C}^{2}-\beta-\mathrm{H}\right), 3.79\left(\mathrm{~s}, 3 \mathrm{H}, 22-\mathrm{NOCH}_{3}\right), 3.81(\mathrm{~s}$, $\left.3 \mathrm{H}, 3-\mathrm{NOCH}_{3}\right), 3.83 \sim 3.80\left(\mathrm{~m}, 1 \mathrm{H}, \mathrm{C}^{6}-\mathrm{H}\right), 7.18(\mathrm{~d}, J=8.1$ $\left.\mathrm{Hz}, 1 \mathrm{H}, \mathrm{C}^{22}-\mathrm{H}\right) ;{ }^{13} \mathrm{C}$ NMR $\left(75 \mathrm{MHz}, \mathrm{CDCl}_{3}\right) \delta: 160.2$ (3-C), 155.5 (22-C), 70.9 (6-C), $61.1\left(22-\mathrm{NOCH}_{3}\right), 61.0$ $\left(3-\mathrm{OCH}_{3}\right), 55.9$ (14-C), 53.9 (17-C), 49.0 (9-C), 42.9 (5-C), 42.8 (13-C), 39.8 (7-C), 39.6 (12-C), 38.9 (10-C), 37.1 (8-C), 36.1 (20-C), 31.7 (4-C), 30.2 (2-C), 27.6 (1-C), 24.3 (16-C), 21.2 (15-C), 21.0 (11-C), 18.1 (21-C), 14.8 (19-C), 12.4 (18-C); IR (KBr) v: 3440, 2945, 2843, 1629, $1560,1417 \mathrm{~cm}^{-1}$.
3.1.5 (3E, 6E, 22E)-22-降-6-羟亚胺基-豆甾-3,22-二 甲氧基肪(5)的合成

$100 \mathrm{mg}(0.20 \mathrm{mmol})$ 化合物 3 溶于 $15 \mathrm{~mL} 95 \%$ 乙醇, 然后加入三水乙酸钠 $34 \mathrm{mg}(0.24 \mathrm{mmol})$, 加热搅拌溶 解, 溶解后一次性加入盐酸羟胺 $26 \mathrm{mg}(0.38 \mathrm{mmol})$, 控 制反应温度在 $65{ }^{\circ} \mathrm{C}$ 左右, TLC 跟踪反应, $2 \mathrm{~h}$ 后停止反 应. 减压抽除大部分乙醇, 出现大量白色固体, 然后加 入少许蒸馏水, 有白色沉淀生成, 随即加入乙酸乙酯溶 解, 用乙酸乙酯萃取 $(20 \mathrm{~mL} \times 3)$, 合并有机层. 有机层 依次用水、饱和食盐水各洗 1 次, 无水硫酸钠干燥. 过 滤, 减压抽除溶剂, 得到无色油状物, 采用柱层析分离 [洗脱剂: $V$ (石油醚) $: V$ (乙酸乙酯 $)=6: 1$ ]得到白色固体 化合物 $551 \mathrm{mg}$, 产率 49.3.\%. m.p. $236 \sim 238{ }^{\circ} \mathrm{C} ;{ }^{1} \mathrm{H}$ NMR (300 MHz, $\left.\mathrm{CDCl}_{3}\right) \delta: 0.71\left(\mathrm{~s}, 3 \mathrm{H}, 18-\mathrm{CH}_{3}\right), 0.86$ (s, $\left.3 \mathrm{H}, 19-\mathrm{CH}_{3}\right), 1.12\left(\mathrm{~d}, J=6.6 \mathrm{~Hz}, 3 \mathrm{H}, 21-\mathrm{CH}_{3}\right), 2.41 \sim 2.28$ (m, $\left.1 \mathrm{H}, \mathrm{C}^{20}-\mathrm{H}\right), 2.70\left(\right.$ br d, $\left.J=13.8 \mathrm{~Hz}, 1 \mathrm{H}, \mathrm{C}^{4}-\mathrm{H}\right), 3.20 \sim$ $3.11\left(\mathrm{~m}, 1 \mathrm{H}, \mathrm{C}^{2}-\beta-\mathrm{H}\right), 3.35(\mathrm{dd}, J=13.8,4.2 \mathrm{~Hz}, 1 \mathrm{H}$, $\left.\mathrm{C}^{7}-\alpha-\mathrm{H}\right), 3.81$ (s, 3H, 3-NOCH$\left.{ }_{3}\right) 3.85$ (s, 3H, 22- $\mathrm{NOCH}_{3}$ ), 7.19 (d, $\left.J=8.4 \mathrm{~Hz}, 1 \mathrm{H}, \mathrm{C}^{22}-\mathrm{H}\right), 9.43(\mathrm{~s}, 1 \mathrm{H}, \mathrm{NOH}) ;{ }^{13} \mathrm{C}$ NMR (75 MHz, $\mathrm{CDCl}_{3}$ ) $\delta: 160.6$ (6-C), 158.0 (3-C), 155.4 $(22-\mathrm{C}), 61.2\left(22-\mathrm{NOCH}_{3}\right), 61.0\left(3-\mathrm{NOCH}_{3}\right), 56.5$ (14-C), 53.9 (17-C), 50.9 (9-C), 44.4(13-C), 43.1 (5-C), 43.0 (10-C), 39.4 (12-C), 37.1 (20-C), 36.6 (8-C), 35.2 (2-C), 29.7 (7-C), 29.3(1-C), 28.2 (16-C), 27.6 (4-C), 24.1 (15-C), 21.3 (11-C), 20.8 (19-C), 18.1 (19-C), 11.8 (18-C); IR (KBr) v: 3411, 2933, 2868, 1643, 1458, 1384, 1045, $906 \mathrm{~cm}^{-1}$; HREIMS calcd for $\mathrm{C}_{24} \mathrm{H}_{40} \mathrm{~N}_{3} \mathrm{O}_{3}[\mathrm{M}+\mathrm{H}]^{+}$ 418.3070 , found 418.3058 .

\section{1 .6 22-降-6-氧代豆留-3, 22-二芐氧基肪(6)的合成}

将 $100 \mathrm{mg}(0.29 \mathrm{mmol})$ 化合物 2 溶于 $20 \mathrm{~mL} 95 \%$ 乙 醇, 然后加入三水乙酸钠 $84 \mathrm{mg}(0.61 \mathrm{mmol})$, 加热搅拌 溶解，待溶解后分批缓慢加入芐氧胺盐酸盐 $88 \mathrm{mg}(0.61$ $\mathrm{mmol}$ ), 约 $10 \mathrm{~min}$ 内加完, 控制反应温度在 $65{ }^{\circ} \mathrm{C}$ 左右, TLC 跟踪反应， $1 \mathrm{~h}$ 后停止反应. 减压蒸去大部分乙醇, 出现大量白色固体, 然后加入少许蒸馏水, 有白色沉淀 生成. 用乙酸乙酯萃取 3 次 $(20 \mathrm{~mL} \times 3)$, 合并有机层, 有 机层依次用水洗，饱和食盐水各洗 1 次，然后用无水硫 酸钠干燥. 过滤, 减压蒸去溶剂, 得到白色固体. 将此 固体采用柱层析分离[洗脱剂: $V$ (石油醚)： $V$ (乙酸乙酯) $=6:$ 1]得到化合物 $664 \mathrm{mg}$, 产率 39.8\%. m.p. 42 43 ${ }^{\circ} \mathrm{C}$; 化合物 6 是由 $(3 E, 22 E)$-及 $(3 Z, 22 E)$-异构体组成的混 合物, 比例为 $1: 1$ ( ${ }^{1} \mathrm{H}$ NMR 确定). ${ }^{1} \mathrm{H}$ NMR $\left(\mathrm{CDCl}_{3}, 300\right.$ $\mathrm{MHz}) \delta: 0.72\left(\mathrm{~s}, 18-\mathrm{CH}_{3}\right), 0.85\left(\mathrm{~s}, 19-\mathrm{CH}_{3}\right), 1.14(\mathrm{~d}, J=$ $\left.6.6 \mathrm{~Hz}, 3 \mathrm{H}, 21-\mathrm{CH}_{3}\right), 3.26 \sim 3.28\left(\mathrm{~m}, 0.5 \mathrm{H}, \mathrm{C}^{2}-\beta-\mathrm{H}, Z\right)$, $3.33 \sim 3.31\left(\mathrm{~m}, 0.5 \mathrm{H}, \mathrm{C}^{4}-\beta-\mathrm{H}, E\right), 5.07(\mathrm{~s}, 2 \mathrm{H}, 3-\mathrm{N}=$ 
$\left.\mathrm{OCH}_{2} \mathrm{Ph}\right), 5.08\left(\mathrm{~d}, J=2.7 \mathrm{~Hz}, 2 \mathrm{H}, 22-\mathrm{N}=\mathrm{OCH}_{2} \mathrm{Ph}\right), 7.35$ $\left(\mathrm{t}, J=3.0 \mathrm{~Hz}, 1 \mathrm{H}, \mathrm{C}^{22}-\mathrm{H}\right), 7.38 \sim 7.28(\mathrm{~m}, 10 \mathrm{H}$, 3,22- $\left.\mathrm{C}_{6} \mathrm{H}_{5}\right) ;{ }^{13} \mathrm{C}$ NMR $\left(75 \mathrm{MHz}, \mathrm{CDCl}_{3}\right) \delta: 12.4$ (18-C), 18.1 (19-C), 20.7 (21-C), 21.3 (11-C), 21.4 (15-C), 24.0 (16-C), 26.9 (1-C), 27.2 (2-C), 27.4 (4-C), 37.1 (20-C), 37.9 (10-C), 39.1 (8-C), 41.8 (12-C), 43.2 (7-C), 46.6 (13-C), 53.6 (9-C), 53.8 (17-C), 56.5 (5-C, E), 56.7 (5-C, $\mathrm{Z}$ ), 58.0 (14-C), 75.3 (3-OCH $2, \mathrm{Z}$-isomer), 75.4 (3- $\mathrm{OCH}_{2}$, $E$-isomer), $75.5\left(22-\mathrm{OCH}_{2}\right),[127.7,127.8,128.0,128.2$, $128.3,128.4,137.7,138.0,138.1]\left(3,22-\mathrm{C}_{6} \mathrm{H}_{5}\right), 155.8$ (22-C), 158.9 (3-C, Z), 159.1 (3-C, E), 210.0 (6-C). IR (KBr) $v$ : 3427, 2941, 2864, 2357, 1711, 1629, 1458, 1376, $1082,1049,918,694 \mathrm{~cm}^{-1}$.

\subsection{2-降-6-羟基豆甾-3,22-二華氧基肪(7)的合成}

室温下将 $175 \mathrm{mg}(0.32 \mathrm{mmol})$ 化合物 6 溶解于 20 $\mathrm{mL}$ 无水甲醇中(加入约 $2 \mathrm{~mL}$ 二氯甲烷助溶), 待溶解完 全后, $20 \mathrm{~min}$ 内分批加入 $24 \mathrm{mg}(0.64 \mathrm{mmol}) \mathrm{NaBH}_{4}$. $\mathrm{TLC}$ 监测反应, 无原料点后即用 $1 \mathrm{~mol} / \mathrm{L} \mathrm{HCl}$ 淬灭反应, 减压蒸去大部分甲醇, 用乙酸乙酯萃取 $(20 \mathrm{~mL} \times 3)$, 合 并有机层. 有机层用水、饱和食盐水各洗 1 次, 无水硫 酸钠干燥. 减压蒸去溶剂, 得到白色固体, 将此固体采 用柱层析分离 [洗脱剂: $V$ (石油醚) $: V$ ( 乙酸乙酯 $)=6:$ 1 ], 得到化合物 $(3 E, 22 E)$-22-降-6-羟基豆甾-3,22-二芐氧 基肜(7a) $71 \mathrm{mg}$, 产率 40.3\%. m.p. $118 \sim 119{ }^{\circ} \mathrm{C} ;{ }^{1} \mathrm{H}$ NMR $\left(\mathrm{CDCl}_{3}, 300 \mathrm{MHz}\right) \delta: 0.75\left(\mathrm{~s}, 18-\mathrm{CH}_{3}\right), 1.13\left(\mathrm{~s}, 19-\mathrm{CH}_{3}\right)$, $1.14\left(\mathrm{~d}, J=6.0 \mathrm{~Hz}, 3 \mathrm{H}, 21-\mathrm{CH}_{3}\right), 2.12(\mathrm{dd}, J=14.7,3.0$ $\left.\mathrm{Hz}, 1 \mathrm{H}, \mathrm{C}_{4}-\beta-\mathrm{H}\right), 2.45 \sim 2.35\left(\mathrm{~m}, 1 \mathrm{H}, \mathrm{C}_{20}-\mathrm{H}\right), 2.57(\mathrm{t}, J=$ $\left.14.1 \mathrm{~Hz}, 1 \mathrm{H}, \mathrm{C}_{4}-\alpha-\mathrm{H}\right), 3.26(\mathrm{dt}, J=15.3,3.0 \mathrm{~Hz}, 1 \mathrm{H}$, $\left.\mathrm{C}_{2}-\beta-\mathrm{H}\right), \quad 3.80 \sim 3.87\left(\mathrm{~m}, 1 \mathrm{H}, \quad \mathrm{C}_{6}-\mathrm{H}\right), \quad 5.07 \quad(\mathrm{~s}, \quad 2 \mathrm{H}$, $\left.22-\mathrm{NOCH}_{2}\right), 5.084\left(\mathrm{~s}, 2 \mathrm{H}, 3-\mathrm{NOCH}_{2}\right), 7.38 \sim 7.28(\mathrm{~m}$, $\left.10 \mathrm{H}, \mathrm{C}_{6} \mathrm{H}_{5} \times 2\right), 7.38\left(\mathrm{~d}, J=4.5 \mathrm{~Hz}, 1 \mathrm{H}, \mathrm{C}^{22}-\mathrm{H}\right) ;{ }^{13} \mathrm{C} \mathrm{NMR}$ $\left(75 \mathrm{MHz}, \mathrm{CDCl}_{3}\right) \delta: 160.8$ (3-C), 156.2 (22-C), [138.2, $137.8,128.4,128.4,128.3,128.3,128.2,128.2,127.9$, 127.9, 127.8, 127.6] $\left(\mathrm{C}_{6} \mathrm{H}_{5} \times 2\right), 75.4\left(3-\mathrm{OCH}_{2}\right), 75.2$ $\left(22-\mathrm{OCH}_{2}\right), 71.0$ (6-C), 55.9 (14-C), 53.94 (17-C), 53.88 (9-C), 49.0 (5-C), 42.9 (7-C), 39.7 (13-C), 39.6 (10-C), 38.9 (12-C), 37.2 (8-C), 36.1 (20-C), 31.7 (4-C), 30.2 (2-C), 27.6 (16-C), 24.3 (15-C), 21.5 (1-C), 21.0 (11-C), 18.1 (21-C), 14.9 (19-C), 12.4 (18-C). IR (KBr) v: 3440, 2929, 1629, 1552, 1413, 1017, $698 \mathrm{~cm}^{-1}$; HREIMS calcd for $\mathrm{C}_{36} \mathrm{H}_{49} \mathrm{~N}_{2} \mathrm{O}_{3} \quad[\mathrm{M}+\mathrm{H}]^{+}$557.3743, found 557.3741.

另外, 分离得到 7 $\mathbf{a}$ 的异构体 22-降-(3Z,22E)-6-差基 豆甾-3,22-二苠氧基肜(7b): $52 \mathrm{mg}$, 产率 29.6\%. m.p. $60 \sim 61{ }^{\circ} \mathrm{C} ;{ }^{1} \mathrm{H}$ NMR $\left(\mathrm{CDCl}_{3}, 300 \mathrm{MHz}\right) \delta: 0.75(\mathrm{~s}$, $\left.18-\mathrm{CH}_{3}\right), 1.11\left(\mathrm{~s}, 19-\mathrm{CH}_{3}\right), 1.13(\mathrm{~d}, J=6.6 \mathrm{~Hz}, 3 \mathrm{H}$,
$\left.21-\mathrm{CH}_{3}\right), 2.44 \sim 2.34\left(\mathrm{~m}, 1 \mathrm{H}, \mathrm{C}^{20}-\mathrm{H}\right), 3.05(\mathrm{dd}, J=15.0$, $\left.3.0 \mathrm{~Hz}, 1 \mathrm{H}, \mathrm{C}^{4}-\beta-\mathrm{H}\right), 3.86 \sim 3.80\left(\mathrm{~m}, 1 \mathrm{H}, \mathrm{C}^{6}-\mathrm{H}\right), 5.06(\mathrm{~s}$, $\left.2 \mathrm{H}, 22-\mathrm{NOCH}_{2}\right), 5.09$ (s, 2H, 3-NOCH 2$), 7.38 \sim 7.29(\mathrm{~m}$, $\left.10 \mathrm{H}, 2 \times \mathrm{C}_{6} \mathrm{H}_{5}\right), 7.37\left(\mathrm{~d}, J=5.1 \mathrm{~Hz}, 1 \mathrm{H}, \mathrm{C}^{22}-\mathrm{H}\right) ;{ }^{13} \mathrm{C} \mathrm{NMR}$ $\left(75 \mathrm{MHz}, \mathrm{CDCl}_{3}\right) \delta: 160.8$ (3-C), 156.2 (22-C), [138.2, $137.8,128.4,128.4,128.3,128.3,128.2,128.2,128.0$, 128.0, 127.7, 127.6] $\left(\mathrm{C}_{6} \mathrm{H}_{5}\right), 75.4 \quad\left(3-\mathrm{NOCH}_{2}\right), 75.2$ (22- $\left.\mathrm{NOCH}_{2}\right), 71.0$ (6-C), 55.9 (14-C), 53.94 (17-C), 53.90 (9-C), 47.7 (5-C), 42.9 (7-C), 39.8 (13-C), 39.7 (10-C), 39.6 (12-C), 37.2 (8-C), 36.1 (20-C), 30.2 (4-C), 27.8 (2-C), 27.6 (16-C), 25.5 (1-C), 24.3 (15-C), 20.9 (11-C), 18.1 (21-C), 14.9 (19-C), 12.4 (18-C); IR (KBr) v: 3423, 2361, 2340, 1634, 1560,1417, 1119, 1037, $1021 \mathrm{~cm}^{-1}$; HREIMS calcd for $\mathrm{C}_{36} \mathrm{H}_{49} \mathrm{~N}_{2} \mathrm{O}_{2}[\mathrm{M}+\mathrm{H}]^{+}$557.3743, found 557.3753 .

\section{$3.1 .83 \beta$-乙酰氧基豆甾-6-䣳(9)的合成}

$100 \mathrm{mg}(0.93 \mathrm{mmol})$ 化合物 8 溶于 $4 \mathrm{~mL}$ 吡啶中, 待 溶解完全后, 慢慢滴加 $0.4 \mathrm{~mL}$ 乙酸䣶, 继续摚拌 30 $\min$, 然后将反应物于室温下静置 $24 \mathrm{~h}$. 加蒸馏水 15 $\mathrm{mL}$, 用乙酸乙酯萃取 $(15 \mathrm{~mL} \times 3)$, 有机层依次用 1 $\mathrm{mol} / \mathrm{L}$ 盐酸, 饱和碳酸氢钠溶液, 水各洗 1 次, 最后用饱 和食盐水洗至中性, 无水硫酸钠干燥. 过滤, 减压蒸出 溶剂, 得到白色固体, 柱层析分离[流动相: $V$ (乙酸乙 酯)： $V$ (石油醚) $=1 ： 8$ ]得白色固体 $989 \mathrm{mg}$, 产率 81.2\%. m.p 138 $\sim 140{ }^{\circ} \mathrm{C}$ (lit. ${ }^{[16]}: 146 \sim 147{ }^{\circ} \mathrm{C}$ ); ${ }^{1} \mathrm{H}$ NMR $\left(\mathrm{CDCl}_{3}, 300 \mathrm{MHz}\right) \delta: 0.69\left(\mathrm{~s}, 3 \mathrm{H}, 18-\mathrm{CH}_{3}\right), 0.78(\mathrm{~s}, 3 \mathrm{H}$, $\left.19-\mathrm{CH}_{3}\right), 0.80$ (d, $J=6.6 \mathrm{~Hz}, 3 \mathrm{H}, 26-\mathrm{CH}_{3}$ or $\left.27-\mathrm{CH}_{3}\right), 0.82$ (t, $J=7.5 \mathrm{~Hz}, 3 \mathrm{H}, 26-\mathrm{CH}_{3}$ or $\left.27-\mathrm{CH}_{3}\right), 0.80(\mathrm{~d}, J=6.6 \mathrm{~Hz}$, $3 \mathrm{H}, 26-\mathrm{CH}_{3}$ or $\left.27-\mathrm{CH}_{3}\right), 1.03\left(\mathrm{~d}, J=6.6 \mathrm{~Hz}, 3 \mathrm{H}, 21-\mathrm{CH}_{3}\right)$, $2.04\left(\mathrm{~s}, 3 \mathrm{H}, 3-\mathrm{CH}_{3} \mathrm{CO}\right), 2.36 \sim 2.25\left(\mathrm{~m}, 2 \mathrm{H}, 7-\mathrm{CH}_{2}\right)$, $4.74 \sim 4.63\left(\mathrm{~m}, 1 \mathrm{H}, \mathrm{C}^{3}-\alpha-\mathrm{H}\right), 5.03(\mathrm{dd}, J=15.3,8.4 \mathrm{~Hz}$, $\left.1 \mathrm{H}, \mathrm{C}^{22}-\mathrm{H}\right), 5.16\left(\mathrm{dd}, J=15.3,8.4 \mathrm{~Hz}, 1 \mathrm{H}, \mathrm{C}^{23}-\mathrm{H}\right) ;{ }^{13} \mathrm{C}$ $\mathrm{NMR}\left(\mathrm{CDCl}_{3}, 75 \mathrm{MHz}\right) \delta: 210.4$ (6-C), $170.6\left(3-\mathrm{COCH}_{3}\right)$, 138.0 (22-C), 129.6 (23-C), 72.8 (3-C), 56.8 (14-C), 56.5 (17-C), 55.9 (5-C), 53.8 (9-C), 51.2 (24-C), 46.7 (7-C), 42.9 (13-C), 41.0 (20-C), 40.4 (12-C), 39.3 (10-C), 37.9 (8-C), 36.4 (1-C), 31.9 (25-C), 28.7 (16-C), 26.8 (2-C), 26.1 (28-C), 25.4 (4-C), 24.0 (15-C), 21.5 (11-C), 21.3 (3- $\left.\mathrm{CH}_{3} \mathrm{CO}\right), 21.2$ (27-C), 21.1 (26-C), 19.0 (21-C), 13.0 (19-C), 12.3 (18-C), 12.2 (29-C); IR (KBr) v: 2958, 2872, 1740, 1707, 1466, 1384, 1240, 1025, $976 \mathrm{~cm}^{-1}$.

3.1 . 22-降-3 $\beta$-乙酰氧基豆甾-6-酮-22-醛(10)的合成

制备方法与化合物 2 类似, 以化合物 9 为原料, 产 率 74.1\%. 白色固体, m.p 154 156 ${ }^{\circ} \mathrm{C} ;{ }^{1} \mathrm{H} \mathrm{NMR}\left(\mathrm{CDCl}_{3}\right.$, $300 \mathrm{MHz}) \delta: 0.73$ (s, 3H, 18- $\left.\mathrm{CH}_{3}\right), 0.80$ (s, 3H, 19- $\mathrm{CH}_{3}$ ), 
$1.15\left(\mathrm{~d}, J=6.6 \mathrm{~Hz}, 3 \mathrm{H}, 21-\mathrm{CH}_{3}\right), 2.05\left(\mathrm{~s}, 3 \mathrm{H}, 3-\mathrm{CH}_{3} \mathrm{CO}\right.$ ), $2.37 \sim 2.26\left(\mathrm{~m}, 2 \mathrm{H}, 7-\mathrm{CH}_{2}\right), 4.74 \sim 4.63\left(\mathrm{~m}, 1 \mathrm{H}, \mathrm{C}^{3}-\alpha-\mathrm{H}\right)$, $9.60\left(\mathrm{~d}, J=3.0 \mathrm{~Hz}, 1 \mathrm{H}, \mathrm{C}^{22}-\mathrm{H}\right) ;{ }^{13} \mathrm{C} \mathrm{NMR}\left(\mathrm{CDCl}_{3}, 75\right.$ $\mathrm{MHz}) \delta: 210.0$ (6-C), 204.7 (22-C), $170.6\left(3-\mathrm{COCH}_{3}\right)$, 72.8 (3-C), 56.5 (14-C), 55.9 (5-C), 53.8 (9-C), 50.9 (17-C), 49.4 (20-C), 46.5 (7-C), 43.5 (13-C), 41.0 (12-C), 39.1 (10-C), 37.8 (8-C), 36.4 (1-C), 26.9 (2-C), 26.8 (4-C), 26.1 (16-C), 24.3 (15-C), $21.4\left(3-\mathrm{CH}_{3} \mathrm{CO}\right), 21.3$ (11-C), 13.4 (19-C), 13.0 (18-C), 12.4 (21-C); IR (KBr) v: 2937, 2880, 2831, 1723, 1707, 1446, 1384, 1241, $1070 \mathrm{~cm}^{-1}$.

3.1.10 22-降-3 $\beta$-乙酰氧基豆甾-6-酮-22-芐氧基肪 (11)的合成

制备程序与化合物 $\mathbf{6}$ 类似, 但是以化合物 10 为原 料, 化合物 10 与芐氧胺盐酸盐的投料摩尔比为 $1: 1$, 产率 70.6\%. 得到白色固体. m.p. $100 \sim 102{ }^{\circ} \mathrm{C} ;{ }^{1} \mathrm{H}$ NMR $\left(300 \mathrm{MHz}, \mathrm{CDCl}_{3}\right) \delta: 0.68\left(18-\mathrm{CH}_{3}\right), 0.764\left(19-\mathrm{CH}_{3}\right), 1.11$ (d, $\left.J=6.6 \mathrm{~Hz}, 1 \mathrm{H}, 21-\mathrm{CH}_{3}\right), 2.02\left(\mathrm{~s}, 3 \mathrm{H}, 3-\mathrm{COCH}_{3}\right)$, $2.33 \sim 2.23\left(\mathrm{~m}, 2 \mathrm{H}, \mathrm{C}^{7}-\mathrm{H}\right), 2.42 \sim 2.32\left(\mathrm{~m}, 1 \mathrm{H}, \mathrm{C}^{20}-\mathrm{H}\right)$, $4.72 \sim 4.61\left(\mathrm{~m}, 1 \mathrm{H}, \mathrm{C}^{3}-\alpha-\mathrm{H}\right), 5.04\left(\mathrm{~s}, 2 \mathrm{H}, \mathrm{OCH}_{2} \mathrm{Ph}\right), 7.27$ (d, $\left.J=8.1 \mathrm{~Hz}, 1 \mathrm{H}, \mathrm{C}^{22}-\mathrm{H}\right), 7.35 \sim 7.29\left(\mathrm{~m}, 5 \mathrm{H}, \mathrm{C}_{6} \mathrm{H}_{5}\right) ;{ }^{13} \mathrm{C}$ NMR (75 MHz, $\left.\mathrm{CDCl}_{3}\right) \delta: 210.1$ (6-C), $170.6\left(\mathrm{OCOCH}_{3}\right)$, 155.8 (22-C), [127.8, 128.1, 128.1, 128.4, 128.4, 137.7] $\left(\mathrm{C}_{6} \mathrm{H}_{5}\right), 75.4\left(22-\mathrm{NOCH}_{2}\right), 72.8$ (3-C), $56.4(5-\mathrm{C}), 53.7$ (14-C), 53.7 (17-C), 46.5 (13-C), 43.2 (9-C), 43.2 (7-C), 40.9 (10-C), 39.1 (12-C), 37.8 (8-C), 37.1 (20-C), 36.4 (1-C), 27.4 (2-C), 26.8 (4-C), 26.1 (16-C), 24.0 (15-C), $21.4\left(3-\mathrm{CH}_{3} \mathrm{CO}\right), 21.3$ (11-C), 18.1 (21-C), 13.0 (19-C), 12.3 (18-C); IR (KBr) v: 3432, 2925, 2859, 2353, 1634, 1380, 1057, 882, 694, $600 \mathrm{~cm}^{-1}$; HREIMS calcd for $\mathrm{C}_{31} \mathrm{H}_{44} \mathrm{NO}_{4}[\mathrm{M}+\mathrm{H}]^{+} \quad 494.3270$, found 494.3283 .

3.1.11 22-降-3 $\beta$-乙酰氧基豆甾-6-羟亚胺基-22-華氧 基肪(12)的合成

将 $100 \mathrm{mg}(0.20 \mathrm{mmol})$ 化合物 $\mathbf{1 1}$ 溶于 $20 \mathrm{~mL} 95 \%$ 乙 醇, 然后加入三水乙酸钠 $27 \mathrm{mg}(0.20 \mathrm{mmol})$, 加热搅拌 溶解, 待溶解后, 分批缓慢加入盐酸着胺 $21 \mathrm{mg}(0.30$ $\mathrm{mmol}$ ), 约 $10 \mathrm{~min}$ 内加完, 控制反应温度在 $65{ }^{\circ} \mathrm{C}$ 左右, TLC 跟踪反应, $3 \mathrm{~h}$ 后停止反应, 减压蒸去大部分乙醇, 出现大量白色固体. 然后加入少许蒸馏水, 有白色沉淀 生成, 用乙酸乙酯萃取 $(20 \mathrm{~mL} \times 3)$, 合并有机层, 有机 层依次用水洗, 饱和食盐水各洗 1 次, 然后用无水硫酸 钠干燥. 过滤, 减压蒸去溶剂, 得到白色固体. 将此固 体采用柱层析分离[洗脱剂: $V$ (石油醚) $: V$ (乙酸乙酯 $)=$ 6：1]得到化合物 $1268 \mathrm{mg}$, 产率 66.0\%. m.p. 140 141 ${ }^{\circ} \mathrm{C} ;{ }^{1} \mathrm{H}$ NMR $\left(300 \mathrm{MHz}, \mathrm{CDCl}_{3}\right) \delta: 0.69$ (s, $3 \mathrm{H}, 18-\mathrm{CH}_{3}$ ), $0.79\left(\mathrm{~s}, 3 \mathrm{H}, 19-\mathrm{CH}_{3}\right) 1.12\left(\mathrm{~d}, J=6.6 \mathrm{~Hz}, 3 \mathrm{H}, 21-\mathrm{CH}_{3}\right)$,
2.05 (s, 3H, 3- $\mathrm{CH}_{3} \mathrm{CO}$ ), $2.45 \sim 2.33\left(\mathrm{~m}, 1 \mathrm{H}, \mathrm{C}^{20}-\mathrm{H}\right), 3.34$ (dd, $\left.J=13.5,4.5 \mathrm{~Hz}, 1 \mathrm{H}, \mathrm{C}^{7}-\alpha-\mathrm{H}\right), 4.75 \sim 4.65(\mathrm{~m}, 1 \mathrm{H}$, $\left.\mathrm{C}^{3}-\alpha-\mathrm{H}\right), 5.06\left(\mathrm{~s}, 2 \mathrm{H}, \mathrm{OCH}_{2}\right), 7.28(\mathrm{~d}, J=5.7 \mathrm{~Hz}, 1 \mathrm{H}$, $\left.\mathrm{C}^{22}-\mathrm{H}\right), 7.39 \sim 7.28\left(\mathrm{~m}, 5 \mathrm{H}, \mathrm{C}_{6} \mathrm{H}_{5}\right), 9.07(\mathrm{~s}, 1 \mathrm{H}, \mathrm{NOH}) ;{ }^{13} \mathrm{C}$ NMR $\left(75 \mathrm{MHz}, \mathrm{CDCl}_{3}\right) \delta: 170.7\left(\mathrm{COCH}_{3}\right), 159.3$ (6-C), 156.1 (22-C), [127.8, 128.2, 128.2, 128.4, 128.4, 137.7] $\left(\mathrm{C}_{6} \mathrm{H}_{5}\right), 75.4\left(\mathrm{OCH}_{2}\right), 73.2(3-\mathrm{C}), 56.4$ (14-C), 54.2 (17-C), 53.8 (9-C), 49.3 (13-C), 43.1 (5-C ), 39.3 (10-C), 38.9 (12-C), 37.1 (4-C), 35.9 (1-C), 35.7 (20-C), 29.6 (8-C), 27.6 (7-C), 27.5 (2-C), 27.1 (16-C), 24.1 (15-C), 21.5 (3- $\mathrm{CH}_{3} \mathrm{CO}$ ), 21.4 (11-C), 18.1 (21-C), 12.6 (19-C), 12.4 (18-C); HREIMS calcd for $\mathrm{C}_{31} \mathrm{H}_{45} \mathrm{~N}_{2} \mathrm{O}_{4} \quad[\mathrm{M}+\mathrm{H}]{ }^{+}$ 509.3379, found 509.3381.

3.1.12 22-降-3 $\beta$-羟基豆甾-6-羟亚胺基-22-芐氧基肪 (13)的合成

将化合物 $12100 \mathrm{mg}$ 溶于 $20 \mathrm{~mL} \mathrm{KOH} / \mathrm{CH}_{3} \mathrm{OH}(1$ $\mathrm{mol} / \mathrm{L}$ )中(加入约 $2 \mathrm{~mL}$ 二氯甲烷助溶), 在室温下搅拌反 应, TLC 跟踪反应, $30 \mathrm{~min}$ 后反应停止. 减压蒸去溶剂得 到白色固体, 加入 $20 \mathrm{~mL}$ 水, 用乙酸乙酯萃取 (20 $\mathrm{mL} \times 3)$, 合并有机层并依次用水、饱和食盐水各洗 1 次, 无水硫酸钠干燥. 减压蒸去溶剂得到白色固体. 柱层析 分离[洗脱剂: $V$ (石油醚) $: V$ (乙酸乙酯 $)=1: 1$ ] 得到白色 固体化合物 $1386 \mathrm{mg}$, 产率 $92.9 \%$. m.p. $140 \sim 141{ }^{\circ} \mathrm{C} ;{ }^{1} \mathrm{H}$ NMR (300 MHz, $\left.\mathrm{CDCl}_{3}\right) \delta: 0.67$ (18-C), 0.77 (19-C), 1.11 (d, $\left.J=6.6 \mathrm{~Hz}, 1 \mathrm{H}, 21-\mathrm{CH}_{3}\right), 2.20$ (br s, $\left.1 \mathrm{H}, \mathrm{OH}\right), 2.43 \sim$ $2.32\left(\mathrm{~m}, 1 \mathrm{H}, \mathrm{C}^{20}-\mathrm{H}\right), 3.32(\mathrm{dd}, J=13.5,4.2 \mathrm{~Hz}, 1 \mathrm{H}$, $\left.\mathrm{C}^{7}-\alpha-\mathrm{H}\right), 3.62 \sim 3.55\left(\mathrm{~m}, 1 \mathrm{H}, \mathrm{C}^{3}-\alpha-\mathrm{H}\right), 5.05\left(\mathrm{~s}, 2 \mathrm{H}, \mathrm{OCH}_{2}\right)$, $7.28\left(\mathrm{~d}, J=8.1 \mathrm{~Hz}, 1 \mathrm{H}, \mathrm{C}^{22}-\mathrm{H}\right), 7.37 \sim 7.28\left(\mathrm{~m}, 5 \mathrm{H}, \mathrm{C}_{6} \mathrm{H}_{5}\right)$, 9.39 (br s, $1 \mathrm{H}, \mathrm{NOH}) ;{ }^{13} \mathrm{C} \mathrm{NMR}\left(75 \mathrm{MHz}, \mathrm{CDCl}_{3}\right) \delta: 159.6$ (6-C), 156.1 (22-C), [127.8, 128.2, 128.2, 128.4, 128.4, 137.7] $\left(\mathrm{C}_{6} \mathrm{H}_{5}\right), 75.4\left(\mathrm{OCH}_{2}\right), 71.1$ (3-C), 56.5 (14-C), 54.2 (17-C), 53.8 (9-C), 49.5 (13-C), 43.1 (5-C), 39.4 (10-C), 38.9 (12-C), 37.2 (4-C), 36.2 (1-C), 35.6 (20-C), 31.5 (8-C), 30.7 (2-C), 29.6 (7-C), 27.6 (16-C), 24.2 (15-C), 21.4 (11-C), 18.1 (21-C), 12.6 (19-C), 12.4 (18-C); IR (KBr) $v$ : 3407, 2937, 2868, 1650, 1454, 1376, 1254, 1200, 1049, 972, $910 \mathrm{~cm}^{-1}$; ESI-MS m/z: $467.3(\mathrm{M}+\mathrm{H})^{+}$. HREIMS calcd for $\mathrm{C}_{29} \mathrm{H}_{43} \mathrm{~N}_{2} \mathrm{O}_{3}[\mathrm{M}+\mathrm{H}]^{+}$467.3274, found 467.3271.

3.1.13 22-降-3 $\beta$-乙酰氧基豆甾-22-華氧肜基-6-缩胺 硫腙(14)的合成

$100 \mathrm{mg}(0.20 \mathrm{mmol})$ 化合物 $\mathbf{1 1}$ 置于 $100 \mathrm{~mL}$ 反应瓶 中, 加入 $30 \mathrm{~mL}$ 无水乙醇, 滴入 $2 \sim 3$ 滴冰醋酸控制溶 液 $\mathrm{pH}=3 \sim 5$, 在 $80{ }^{\circ} \mathrm{C}$ 搅拌溶解, 加入 $36 \mathrm{mg}(0.40$ $\mathrm{mmol}$ )硫代氨基脲, TLC 板跟踪反应. 约 $2 \mathrm{~h}$ 后没有原料 
点停止反应, 减压蒸去大部分乙醇, 得到淡黄色固体. 加入 $20 \mathrm{~mL}$ 水, 用乙酸乙酯萃取 $(20 \mathrm{~mL} \times 3)$, 合并有机 层并依次用水、饱和食盐水各洗 1 次, 无水硫酸钠干燥. 减压蒸去溶剂得到淡黄色固体. 柱层析分离[洗脱剂: $V$ (二氯甲烷) $: V$ (甲醇) $=80 ：$ ] 得到化合物 $\mathbf{1 4} 81 \mathrm{mg}$, 产率 70.6\%. m.p. $107 \sim 108{ }^{\circ} \mathrm{C} ;{ }^{1} \mathrm{H}$ NMR $(300 \mathrm{MHz}$, $\left.\mathrm{CDCl}_{3}\right) \delta: 0.64\left(\mathrm{~s}, 3 \mathrm{H}, 18-\mathrm{CH}_{3}\right), 0.68\left(\mathrm{~s}, 3 \mathrm{H}, 19-\mathrm{CH}_{3}\right), 1.07$ (d, $J=6.9 \mathrm{~Hz}, 3 \mathrm{H}, 21-\mathrm{CH}_{3}$ ), 2.03 (s, 3H, 3- $\mathrm{CH}_{3} \mathrm{CO}$ ), 2.69 (br d, $\left.J=10.5 \mathrm{~Hz}, 1 \mathrm{H}, \mathrm{C}^{4}-\mathrm{H}\right), 4.72 \sim 4.61\left(\mathrm{~m}, 1 \mathrm{H}, \mathrm{C}^{3}-\alpha-\mathrm{H}\right)$, $5.01\left(\mathrm{~s}, 2 \mathrm{H}, \mathrm{OCH}_{2}\right), 6.91\left(\mathrm{~d}, J=3.9 \mathrm{~Hz}, 1 \mathrm{H}, \mathrm{NH}_{2}\right), 7.18(\mathrm{~d}$, $\left.J=3.9 \mathrm{~Hz}, 1 \mathrm{H}, \mathrm{NH}_{2}\right), 7.25\left(\mathrm{~d}, J=8.1 \mathrm{~Hz}, 1 \mathrm{H}, \mathrm{C}^{22}-\mathrm{H}\right)$, $7.33 \sim 7.25\left(\mathrm{~m}, 5 \mathrm{H}, \mathrm{C}_{6} \mathrm{H}_{5}\right), 9.07(\mathrm{~s}, 1 \mathrm{H}, \mathrm{NH}) ;{ }^{13} \mathrm{C} \mathrm{NMR}(75$ $\left.\mathrm{MHz}, \mathrm{CDCl}_{3}\right) \delta: 178.7(\mathrm{C}=\mathrm{S}), 170.7\left(\mathrm{COCH}_{3}\right), 155.8$ (6-C), 155.4 (22-C), [127.8, 128.2, 128.2, 128.4, 128.4, 137.7] $\left(\mathrm{C}_{6} \mathrm{H}_{5}\right), 75.4\left(\mathrm{OCH}_{2}\right), 73.3$ (3-C), 56.1 (14-C), 54.0 (17-C), 53.7 (9-C), 50.8 (5-C), 43.2 (13-C), 39.5 (12-C), 39.1 (10-C), 37.0 (4-C), 36.3 (1-C), 36.3 (8-C), 32.0 (20-C), 27.9 (7-C), 27.5 (2-C), 27.0 (16-C), 24.2 (15-C), 21.5 (3- $\left.\mathrm{CH}_{3} \mathrm{CO}\right), 21.3$ (11-C), 18.1 (21-C), 12.6 (19-C), 12.4 (18-C); IR (KBr) v: 3419, 2941, 2864, 1723, 1597, $1495,1372,1245,1086,1025 \mathrm{~cm}^{-1}$; HREIMS calcd for $\mathrm{C}_{32} \mathrm{H}_{47} \mathrm{~N}_{4} \mathrm{O}_{3} \mathrm{~S}[\mathrm{M}+\mathrm{H}]^{+}$567.3369, found 567.3384.

3.1.14 22-降-3 $\beta$-差基-22-華氧肜基-豆甾-6-缩胺硫 腙(15)的合成

制备方法与化合物 13 相同, 得到淡黄色固体 $\mathbf{1 5}$, 产率 74.4\%. m.p. $137 \sim 138{ }^{\circ} \mathrm{C} ;{ }^{1} \mathrm{H}$ NMR $(300 \mathrm{MHz}$, $\left.\mathrm{CDCl}_{3}\right) \delta: 0.66\left(\mathrm{~s}, 3 \mathrm{H}, 18-\mathrm{CH}_{3}\right), 0.68\left(\mathrm{~s}, 3 \mathrm{H}, 19-\mathrm{CH}_{3}\right), 1.09$ (d, $\left.J=6.6 \mathrm{~Hz}, 3 \mathrm{H}, 21-\mathrm{CH}_{3}\right), 2.40 \sim 2.32\left(\mathrm{~m}, 1 \mathrm{H}, \mathrm{C}^{20}-\mathrm{H}\right)$, $2.75 \sim 2.61\left(\mathrm{~m}, 1 \mathrm{H}, \mathrm{C}^{5}-\mathrm{H}\right), 3.64 \sim 3.57\left(\mathrm{~m}, 1 \mathrm{H}, \mathrm{C}^{3}-\alpha-\mathrm{H}\right)$, $5.03\left(\mathrm{~s}, 2 \mathrm{H}, \mathrm{OCH}_{2}\right), 7.02$ (br s, $\left.1 \mathrm{H}, \mathrm{NH}_{2}\right), 7.423($ br s, $1 \mathrm{H}$, $\left.\mathrm{NH}_{2}\right), 7.26\left(\mathrm{~d}, J=8.4 \mathrm{~Hz}, 1 \mathrm{H}, \mathrm{C}^{22}-\mathrm{H}\right), 7.35 \sim 7.28(\mathrm{~m}, 5 \mathrm{H}$, $\left.\mathrm{C}_{6} \mathrm{H}_{5}\right), 9.05$ (br s, $\left.1 \mathrm{H}, \mathrm{NH}\right) ;{ }^{13} \mathrm{C} \mathrm{NMR}\left(75 \mathrm{MHz}, \mathrm{CDCl}_{3}\right) \delta$ : $178.4(\mathrm{C}=\mathrm{S}), 155.9$ (6-C), 155.8 (22-C), [127.8, 128.2, $128.2,128.4,128.4,137.6]\left(\mathrm{C}_{6} \mathrm{H}_{5}\right), 75.4\left(\mathrm{OCH}_{2}\right), 70.8$ (3-C), 56.2 (14-C), 54.1 (17-C), 53.7 (9-C), 51.0 (5-C), 43.2 (12-C), 39.5 (13-C), 39.4 (4-C), 39.2 (10-C), 37.1 (1-C), 36.4 (8-C), 31.9 (20-C), 31.6 (7-C), 30.8 (2-C), 27.6 (16-C), 24.3 (15-C), 21.4 (11-C), 18.1 (21-C), 12.7 (19-C), 12.4 (18-C); IR (KBr) v: 3415, 2929, 2847, 1593, 1495, 1376, 1266, 1241, 1151, 1082, 1041, 853, 735, $694 \mathrm{~cm}^{-1}$; HREIMS calcd for $\mathrm{C}_{30} \mathrm{H}_{45} \mathrm{~N}_{4} \mathrm{O}_{2} \mathrm{~S}[\mathrm{M}+\mathrm{H}]^{+}$525.3263, found 525.3265 .

3.1.15 22-降-3 $\beta$-乙酰氧基-6-甲氧肜基-豆甾-22-芐 氧基肪(16)的合成

将 $100 \mathrm{mg}(0.20 \mathrm{mmol})$ 化合物 $\mathbf{1 1}$ 溶于 $30 \mathrm{~mL}$ 95\%乙
醇, 然后加入三水乙酸钠 $27 \mathrm{mg}(0.20 \mathrm{mmol})$, 加热搅拌 溶解, 待溶解后分批缓慢加入甲氧基胺盐酸盐 $33 \mathrm{mg}$ $(0.40 \mathrm{mmol})$, 约 $10 \mathrm{~min}$ 内加完, 控制反应温度在 $65{ }^{\circ} \mathrm{C}$ 左右, TLC 跟踪反应, $2 \mathrm{~h}$ 后停止反应, 减压抽除大部分 乙醇, 出现白色固体, 加入少许蒸馏水, 有白色沉淀生 成, 用乙酸乙酯萃取 $(20 \mathrm{~mL} \times 3)$, 合并有机层, 有机层 依次用水、饱和食盐水各洗 1 次, 用无水硫酸钠干燥. 过 滤, 减压抽除溶剂, 得到白色固体. 将此固体采用柱层 析分离 [洗脱剂: $V$ (石油醚)： $V$ (乙酸乙酯) $=20: 1$ ] 得到 化合物 $1669 \mathrm{mg}$, 产率 64.9\%. m.p. 47 48 ${ }^{\circ} \mathrm{C} ;{ }^{1} \mathrm{H}$ NMR $\left(300 \mathrm{MHz}, \mathrm{CDCl}_{3}\right) \delta: 0.70\left(\mathrm{~s}, 3 \mathrm{H}, 18-\mathrm{CH}_{3}\right), 0.78(\mathrm{~s}, 3 \mathrm{H}$, $\left.19-\mathrm{CH}_{3}\right), 1.11\left(\mathrm{~d}, J=6.9 \mathrm{~Hz}, 3 \mathrm{H}, 21-\mathrm{CH}_{3}\right), 2.04(\mathrm{~s}, 3 \mathrm{H}$, $\left.3-\mathrm{COCH}_{3}\right), 2.40 \sim 2.32\left(\mathrm{~m}, 1 \mathrm{H}, \mathrm{C}^{20}-\mathrm{H}\right), 3.21$ (dd, $J=13.5$, $\left.4.5 \mathrm{~Hz}, 1 \mathrm{H}, \mathrm{C}^{7}-\alpha-\mathrm{H}\right), 3.80\left(\mathrm{~s}, 3 \mathrm{H}, \mathrm{OCH}_{3}\right), 4.72 \sim 4.63(\mathrm{~m}$, $\left.1 \mathrm{H}, \mathrm{C}^{3}-\alpha-\mathrm{H}\right), 5.05\left(\mathrm{~s}, 2 \mathrm{H}, \mathrm{OCH}_{2}\right), 7.18(\mathrm{~d}, J=8.1 \mathrm{~Hz}, 1 \mathrm{H}$, $\left.\mathrm{C}^{22}-\mathrm{H}\right), 7.36 \sim 7.25\left(\mathrm{~m}, 5 \mathrm{H}, \mathrm{C}_{6} \mathrm{H}_{5}\right) ;{ }^{13} \mathrm{C}$ NMR $(75 \mathrm{MHz}$, $\left.\mathrm{CDCl}_{3}\right) \delta: 170.7\left(\mathrm{COCH}_{3}\right), 158.3$ (6-C), 155.3 (22), [127.8, $128.2,128.2,128.4,128.4,137.7]\left(\mathrm{C}_{6} \mathrm{H}_{5}\right), 75.4\left(\mathrm{OCH}_{2}\right)$, 73.5 (3-C), $61.2\left(\mathrm{OCH}_{3}\right), 56.4$ (14-C), $54.2(17-\mathrm{C}), 53.8$ (9-C), 49.2 (13-C), 43.1 (5-C), 39.4 (10-C), 38.7 (12-C), 37.1 (4-C), 36.0 (1-C), 35.6 (20-C), 30.1 (8-C), 27.6 (7-C), 27.0 (2-C), 24.1 (16-C), 21.5 (15-C), $21.4\left(3-\mathrm{CH}_{3} \mathrm{CO}\right)$, 21.3 (11-C), 18.1 (21-C), 12.5 (19-C), 12.4 (18-C); IR (KBr) v: 3444, 3411, 2937, 1732, 1638, 1617, 1450, 1384, 1237, 1053, $600 \mathrm{~cm}^{-1}$; HREIMS calcd for $\mathrm{C}_{32} \mathrm{H}_{46} \mathrm{~N}_{2} \mathrm{O}_{4}$ $[\mathrm{M}+\mathrm{H}]^{+}$523.3536, found 523.3549.

3.1.16 22-降-3 $\beta$-羟基-6-甲氧肜基-豆甾-22-芐氧基肪 (17)的合成

制备方法与化合物 $\mathbf{1 3}$ 相同. 得到白色固体 17, 产 率 75.4\%. m.p. $69 \sim 70{ }^{\circ} \mathrm{C} ;{ }^{1} \mathrm{H}$ NMR $\left(300 \mathrm{MHz}, \mathrm{CDCl}_{3}\right) \delta$ : 0.69 (s, 3H, 18- $\left.\mathrm{CH}_{3}\right), 0.75$ (s, 3H, 19- $\mathrm{CH}_{3}$ ), 1.11 (s, $J=6.6$ $\left.\mathrm{Hz}, 3 \mathrm{H}, 21-\mathrm{CH}_{3}\right), 2.40 \sim 2.30\left(\mathrm{~m}, 1 \mathrm{H}, \mathrm{C}_{20}-\mathrm{H}\right), 3.21(\mathrm{dd}$, $\left.J=13.8,4.5 \mathrm{~Hz}, 1 \mathrm{H}, \mathrm{C}^{7}-\alpha-\mathrm{H}\right), 3.61 \sim 3.51(\mathrm{~m}, 1 \mathrm{H}$, $\left.\mathrm{C}^{3}-\alpha-\mathrm{H}\right), 3.81\left(\mathrm{~s}, 3 \mathrm{H}, \mathrm{OCH}_{3}\right), 5.04\left(\mathrm{~s}, 2 \mathrm{H}, \mathrm{OCH}_{2}\right), 7.18(\mathrm{~d}$, $\left.J=8.1 \mathrm{~Hz}, 1 \mathrm{H}, \mathrm{C}^{22}-\mathrm{H}\right), 7.36 \sim 7.25\left(\mathrm{~m}, 5 \mathrm{H}, \mathrm{C}_{6} \mathrm{H}_{5}\right) ;{ }^{13} \mathrm{C}$ NMR $\left(75 \mathrm{MHz}, \mathrm{CDCl}_{3}\right) \delta: 158.8$ (6-C), 155.4 (22-C), $[127.8,128.2,128.2,128.4,128.4,137.7]\left(\mathrm{C}_{6} \mathrm{H}_{5}\right), 75.4$ $\left(\mathrm{OCH}_{2}\right), 71.1$ (3-C), $61.2\left(\mathrm{OCH}_{3}\right), 56.5(14-\mathrm{C}), 54.3$ (17-C), 53.7 (9-C), 49.4 (13-C), 43.1 (5-C), 39.4 (10-C), 38.7 (12-C), 37.1 (4-C), 36.2 (1-C), 35.7 (20-C), 31.6 (8-C), 30.8 (2-C), 30.2 (7-C), 27.7 (16-C), 24.1 (15-C), 21.4 (11-C), 18.1 (21-C), 12.6 (19-C), 12.4 (18-C); IR (KBr) v: 3432, 2925, 2859, 2353, 1634, 1466, 1380, 1057, 882, 694, $600 \mathrm{~cm}^{-1}$; HREIMS calcd for $\mathrm{C}_{30} \mathrm{H}_{45} \mathrm{~N}_{2} \mathrm{O}_{3}[\mathrm{M}+$ $\mathrm{H}]^{+} 481.3430$, found 481.3431 . 


\section{$3.1 .173 \beta$-羟基胆甾-6-甲氧基肜(19)的合成}

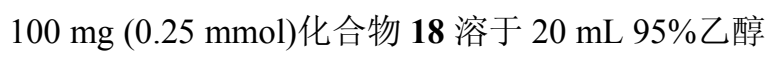
中, 溶解后加入 $34 \mathrm{mg}(0.25 \mathrm{mmol})$ 三水乙酸钠, 保持反 应温度在 $65{ }^{\circ} \mathrm{C}$, 搅拌 $10 \mathrm{~min}$ 后加入 $26 \mathrm{mg}(0.3 \mathrm{mmol})$ 甲氧胺盐酸盐. TLC 监测[展开剂: $V$ (石油醚) $: V$ (乙酸乙 酯 $)=2: 1$ 反反应, 继续反应 $6 \mathrm{~h}$, 无原料点后停止反应. 减压蒸出大部分溶剂, 加入适量水, 用乙酸乙酯萃取, 有机相分别用饱和 $\mathrm{NaHCO}_{3}$ 溶液、水、饱和 $\mathrm{NaCl}$ 溶液 各洗 1 次, 无水硫酸钠干燥. 过滤, 减压蒸去溶剂, 粗产 品经柱层析分离 [洗脱剂: $V$ (石油醚) : $V$ (乙酸乙酯 $)=$ 6：1]得到 $80 \mathrm{mg}$ 白色固体产物 19, 产率 75\%. m.p. $164 \sim 165{ }^{\circ} \mathrm{C} ;{ }^{1} \mathrm{H} \mathrm{NMR}\left(\mathrm{CDCl}_{3}, 300 \mathrm{MHz}\right) \delta: 0.65$ (s, 3H, $\left.18-\mathrm{CH}_{3}\right), 0.75\left(\mathrm{~s}, 3 \mathrm{H}, 19-\mathrm{CH}_{3}\right), 0.86(\mathrm{~d}, J=6.3 \mathrm{~Hz}, 3 \mathrm{H}$, $26-\mathrm{CH}_{3}$ or $\left.27-\mathrm{CH}_{3}\right), 0.87\left(\mathrm{~d}, J=6.3 \mathrm{~Hz}, 3 \mathrm{H}, 26-\mathrm{CH}_{3}\right.$ or $\left.27-\mathrm{CH}_{3}\right), 0.91\left(\mathrm{~d}, J=6.6 \mathrm{~Hz}, 3 \mathrm{H}, 21-\mathrm{CH}_{3}\right), 3.21$ (dd, $J=$ $\left.13.8,4.5 \mathrm{~Hz}, 1 \mathrm{H}, \mathrm{C}^{7}-\alpha-\mathrm{H}\right), 3.62 \sim 3.52\left(\mathrm{~m}, 1 \mathrm{H}, \mathrm{C}^{3}-\alpha-\mathrm{H}\right)$, $3.81\left(\mathrm{~s}, 3 \mathrm{H}, \mathrm{OCH}_{3}\right) ;{ }^{13} \mathrm{C} \mathrm{NMR}\left(\mathrm{CDCl}_{3}, 75 \mathrm{MHz}\right) \delta: 159.0$ (6-C), 71.2 (3-C), $61.1\left(\mathrm{OCH}_{3}\right), 56.6$ (14-C), 56.2 (17-C), 54.3 (9-C), 49.4 (13-C), 42.9 (5-C), 39.7 (10-C), 39.5 (24-C), 38.7 (4-C), 36.2 (12-C), 36.1 (22-C), 35.8 (20-C), 35.7 (1-C), 31.7 (8-C), 30.9 (2-C), 30.2 (16-C), 28.2 (7-C), 28.0 (25-C), 24.1 (23-C), 23.8 (15-C), 22.8 (26-C or 27-C), 22.6 (26-C or 27-C), 21.4 (11-C), 18.6 (21-C), 12.1 (19-C), 12.5 (18-C); IR (KBr) v: 3362, 2937, 2864, 1458, 1380, $1053,976,865 \mathrm{~cm}^{-1}$; HREIMS calcd for $\mathrm{C}_{28} \mathrm{H}_{50} \mathrm{NO}_{2}[\mathrm{M}+$ $\mathrm{H}]^{+}$432.3842, found 432.3818 .

\section{$3.1 .183 \beta$-羟基胆甾-6-芐氧基肪 $(20)$ 的合成}

制备方法与化合物 19 类似, 以苠氧胺盐酸盐代替 甲氧胺盐酸盐, 得到白色固体产物 20, 产率 59\%. m.p. $133 \sim 135{ }^{\circ} \mathrm{C} ;{ }^{1} \mathrm{H}$ NMR $\left(\mathrm{CDCl}_{3}, 300 \mathrm{MHz}\right) \delta: 0.66(\mathrm{~s}, 6 \mathrm{H}$, $18-\mathrm{CH}_{3}$ and $\left.19-\mathrm{CH}_{3}\right), 0.885\left(\mathrm{~d}, J=6.6 \mathrm{~Hz}, 3 \mathrm{H}, 26-\mathrm{CH}_{3}\right.$ or $\left.27-\mathrm{CH}_{3}\right), 0.890\left(\mathrm{~d}, J=6.6 \mathrm{~Hz}, 3 \mathrm{H}, 26-\mathrm{CH}_{3}\right.$ or $\left.27-\mathrm{CH}_{3}\right)$, $0.92\left(\mathrm{~d}, J=6.6 \mathrm{~Hz}, 3 \mathrm{H}, 21-\mathrm{CH}_{3}\right), 3.29(\mathrm{dd}, J=13.3,4.5$ $\left.\mathrm{Hz}, 1 \mathrm{H}, \mathrm{C}^{7}-\alpha-\mathrm{H}\right), 3.61 \sim 3.51\left(\mathrm{~m}, 1 \mathrm{H}, \mathrm{C}^{3}-\alpha-\mathrm{H}\right), 5.08(\mathrm{~s}, 2 \mathrm{H}$, $\left.\mathrm{OCH}_{2}\right), 7.38 \sim 7.26\left(\mathrm{~m}, 5 \mathrm{H}, \mathrm{C}_{6} \mathrm{H}_{5}\right) ;{ }^{13} \mathrm{C} \mathrm{NMR}\left(\mathrm{CDCl}_{3}, 75\right.$ $\mathrm{MHz}) \delta$ : 160.0 (6-C), [138.8, 128.2, 128.2, 128.1, 128.1, 127.5] $\left(\mathrm{C}_{6} \mathrm{H}_{5}\right), 75.2\left(\mathrm{OCH}_{2} \mathrm{Ph}\right), 71.3(3-\mathrm{C}), 56.6$ (14-C), 56.2 (17-C), 54.3 (9-C), 49.6 (5-C), 42.9 (24-C), 39.7 (13-C), 39.5 (4-C), 38.8 (12-C), 36.3 (10-C), 36.1 (22-C), 35.8 (20-C), 35.7 (1-C), 31.6 (8-C), 30.7 (16-C), 30.9 (2-C), 28.2 (7-C), 28.0 (25-C), 24.2 (19-C), 23.8 (15-C), 22.8 (26- or 27-C), 22.6 (26- or 27-C), 21.4 (23-C), 18.7 (11-C), 12.4 (21-C), 12.1 (18-C); IR (KBr) v: 3370, 2945, 1462, 1372, 1049, 856, 739, $702 \mathrm{~cm}^{-1}$; HREIMS calcd for $\mathrm{C}_{34} \mathrm{H}_{54} \mathrm{NO}_{2}[\mathrm{M}+\mathrm{H}]^{+}$508.4155, found 508.4153.

\subsubsection{9 胆甾-6-酮-3-甲氧基肜(22)的合成}

$100 \mathrm{mg}$ (0.25 mmol)胆甾-3,6-二酮(21)溶于 $20 \mathrm{~mL}$ $95 \%$ 乙醇, 溶解完全后加入 $34 \mathrm{mg}(0.25 \mathrm{mmol})$ 三水乙酸 钠, 保持反应温度在 $65{ }^{\circ} \mathrm{C}$; 搅拌 $10 \mathrm{~min}$ 后分批缓慢加 入 $26 \mathrm{mg}(0.3 \mathrm{mmol})$ 甲氧胺盐酸盐. TLC 监测[展开剂: $V($ 石油醚 $): V($ 乙酸乙酯 $)=2: 1]$, 无原料点后停止反 应. 减压蒸出大部分溶剂, 加入适量水, 先用乙酸乙酯 萃取, 有机相再分别用饱和碳酸氢钠溶液、水、饱和食 盐水各洗 1 次, 无水硫酸钠干燥. 减压蒸去溶剂, 粗产 品经柱层析分离 [洗脱剂: $V$ (石油醚) : $V$ (乙酸乙酯 $)=$ 5：1]得到 $75 \mathrm{mg}$ 白色固体产物 22. 产率 70\%. m.p. $122 \sim 124{ }^{\circ} \mathrm{C} ;{ }^{1} \mathrm{H}$ NMR $\left(\mathrm{CDCl}_{3}, 300 \mathrm{MHz}\right) \delta: 0.71(\mathrm{~s}, 3 \mathrm{H}$, $\left.18-\mathrm{CH}_{3}\right), 0.876\left(\mathrm{~d}, J=6.6 \mathrm{~Hz}, 3 \mathrm{H}, 26-\mathrm{CH}_{3}\right.$ or $\left.27-\mathrm{CH}_{3}\right)$, $0.880\left(\mathrm{~d}, J=6.6 \mathrm{~Hz}, 3 \mathrm{H}, 26-\mathrm{CH}_{3}\right.$ or $\left.27-\mathrm{CH}_{3}\right), 0.92(\mathrm{~d}, J=$ $\left.6.6 \mathrm{~Hz}, 3 \mathrm{H}, 21-\mathrm{CH}_{3}\right), 1.12\left(\mathrm{~s}, 3 \mathrm{H}, 19-\mathrm{CH}_{3}\right), 2.01(\mathrm{dt}, J=$ 12.6, 3.2 Hz, 1H, $\mathrm{C}^{8}-\mathrm{H}$ ), 2.09 (br d, $J=14.4 \mathrm{~Hz}, 1 \mathrm{H}$, $\left.\mathrm{C}^{4}-\mathrm{H}\right), 2.56\left(\mathrm{t}, J=13.8 \mathrm{~Hz}, 1 \mathrm{H}, \mathrm{C}^{5}-\mathrm{H}\right), 3.15$ (dd, $J=15.3$, $\left.5.1 \mathrm{~Hz}, 1 \mathrm{H}, \mathrm{C}^{2}-\beta-\mathrm{H}\right), 3.83\left(\mathrm{~s}, 3 \mathrm{H}, \mathrm{OCH}_{3}\right) ;{ }^{13} \mathrm{C} \mathrm{NMR}$ $\left(\mathrm{CDCl}_{3}, 75 \mathrm{MHz}\right) \quad \delta: 210.2$ (6-C), 158.5 (3-C), 61.1 $\left(\mathrm{OCH}_{3}\right), 57.9$ (5-C), 56.8 (14-C), 56.2 (17-C), 56.1 (9-C), 46.7 (13-C), 42.9 (12-C), 39.5 (10-C), 39.0 (24-C), 38.9 (22-C), 36.1 (20-C), 35.7 (8-C), 31.7 (7-C), 31.6 (2-C), 30.2 (16-C), 28.1 (1-C), 28.0 (25-C), 24.8 (4-C), 23.9 (15-C), 23.8 (23-C), 22.8 (27-C), 21.6 (26-C), 21.1 (11-C), 18.7 (21-C), 12.4 (19-C), 12.0 (18-C); IR (KBr) v: 2937, $1703,1458,1380,1057 \mathrm{~cm}^{-1}$; HREIMS calcd for $\mathrm{C}_{28} \mathrm{H}_{48^{-}}$ $\mathrm{NO}_{2}[\mathrm{M}+\mathrm{H}]^{+}$430.3685, found 430.3688.

\section{1 .206 -羟基胆甾-3-甲氧基肟(23)的合成}

制备方法与化合物 4 类似，以化合物 22 为原料，得 到白色固体 23, 产率 $65 \%$. m.p. 163 $165{ }^{\circ} \mathrm{C} ;{ }^{1} \mathrm{H}$ NMR $\left(\mathrm{CDCl}_{3}, 300 \mathrm{MHz}\right) \delta: 0.71\left(\mathrm{~s}, 3 \mathrm{H}, 18-\mathrm{CH}_{3}\right), 0.876(\mathrm{~d}, J=$ $6.6 \mathrm{~Hz}, 3 \mathrm{H}, 26-\mathrm{CH}_{3}$ or $\left.27-\mathrm{CH}_{3}\right), 0.880(\mathrm{~d}, J=6.6 \mathrm{~Hz}, 3 \mathrm{H}$, $26-\mathrm{CH}_{3}$ or $\left.27-\mathrm{CH}_{3}\right), 0.92\left(\mathrm{~d}, J=6.6 \mathrm{~Hz}, 3 \mathrm{H}, 21-\mathrm{CH}_{3}\right), 1.12$ (s, 3H, 19- $\left.\mathrm{CH}_{3}\right), 3.14$ (dd, $\left.J=15.3,5.1 \mathrm{~Hz}, 1 \mathrm{H}, \mathrm{C}^{2}-\beta-\mathrm{H}\right)$, $3.83\left(\mathrm{~s}, 3 \mathrm{H}, \mathrm{OCH}_{3}\right), 3.84\left(\mathrm{~m}, 1 \mathrm{H}, \mathrm{C}^{6}-\mathrm{H}\right) ;{ }^{13} \mathrm{C} \mathrm{NMR}\left(\mathrm{CDCl}_{3}\right.$, $75 \mathrm{MHz}) \delta$ : $160.3(3-\mathrm{C}), 71.2(6-\mathrm{C}), 61.0\left(\mathrm{OCH}_{3}\right), 56.3$ (14-C), 56.1 (17-C), 56.1 (9-C), 49.1 (5-C), 42.7 (13-C), 39.8 (10-C), 39.5 (12-C), 39.0 (24-C), 38.9 (22-C), 36.1 (20-C), 31.7 (2-C), 31.7 (7-C), 30.2 (8-C), 30.2 (16-C), 28.0 (1-C), 28.0 (25-C), 24.3 (4-C), 24.2 (23-C), 23.9 (15-C), 22.8 (27-C), 22.6 (26-C), 21.1 (11-C), 18.7 (21-C), 14.9 (19-C), 12.1 (18-C); IR (KBr) v: 3387, 2921, 2855, 1642, 1462, 1380, $1053 \mathrm{~cm}^{-1}$; HREIMS calcd for $\mathrm{C}_{28} \mathrm{H}_{50} \mathrm{NO}_{2}[\mathrm{M}+\mathrm{H}]^{+}$432.3842, found 432.3852 . 


\section{1 .21 胆甾-3,6-二酮-3-芐氧基肜(24)的合成}

合成方法与化合物 22 相同, 采用茮氧胺盐酸盐代 替甲氧胺盐酸盐. 得到白色固体产物 24, 产率 $61 \%$. m.p. $115 \sim 117{ }^{\circ} \mathrm{C} ;{ }^{1} \mathrm{H} \mathrm{NMR}\left(\mathrm{CDCl}_{3}, 300 \mathrm{MHz}\right) \delta$ : 0.69 (s, $\left.3 \mathrm{H}, 18-\mathrm{CH}_{3}\right), 0.85\left(\mathrm{~s}, 3 \mathrm{H}, 19-\mathrm{CH}_{3}\right), 0.884(\mathrm{~d}, J=6.6 \mathrm{~Hz}$, $3 \mathrm{H}, 26-\mathrm{CH}_{3}$ or $\left.27-\mathrm{CH}_{3}\right), 0.888\left(\mathrm{~d}, J=6.6 \mathrm{~Hz}, 3 \mathrm{H}, 26-\mathrm{CH}_{3}\right.$ or $\left.27-\mathrm{CH}_{3}\right), 0.93\left(\mathrm{~d}, J=6.3 \mathrm{~Hz}, 3 \mathrm{H}, 21-\mathrm{CH}_{3}\right), 2.38 \sim 2.21$ $\left(\mathrm{m}, 4 \mathrm{H}, \mathrm{C}^{4}-\mathrm{H}\right.$ and $\left.\mathrm{C}^{7}-\mathrm{H}\right), 3.28(\mathrm{dd}, J=13.8,3.6 \mathrm{~Hz}, 1 \mathrm{H}$, $\left.\mathrm{C}^{2}-\beta-\mathrm{H}\right), 5.06\left(\mathrm{~s}, 2 \mathrm{H}, \mathrm{OCH}_{2}\right), 7.38 \sim 7.30\left(\mathrm{~m}, 5 \mathrm{H}, \mathrm{C}_{6} \mathrm{H}_{5}\right)$; ${ }^{13} \mathrm{C} \mathrm{NMR}\left(\mathrm{CDCl}_{3}, 75 \mathrm{MHz}\right) \delta: 210.4$ (6-C), 159.2 (3-C), $[127.6,128.0,128.0,128.3,128.3,138.0]\left(\mathrm{C}_{6} \mathrm{H}_{5}\right), 75.3$ $\left(\mathrm{OCH}_{2}\right), 56.7$ (5-C), 56.1 (14-C), 53.7 (17-C), 53.6 (9-C), 46.7 (13-C), 43.0 (7-C), 41.9 (10-C), 41.8 (12-C), 39.5 (24-C), 38.0 (22-C), 37.1 (20-C), 36.1 (8-C), 35.7 (4-C), 28.0 (25-C), 28.0 (16-C), 27.3 (2-C), 24.0 (1-C), 23.8 (15-C), 22.8 (26-C), 22.6 (27-C), 21.5 (23-C), 20.7 (11-C), 18.7 (21-C), 12.5 (19-C), 12.0 (18-C); IR (KBr) v: 3415, 1703, 1466, 1386, 1237, 1041, $914 \mathrm{~cm}^{-1}$; HREIMS calcd for $\mathrm{C}_{34} \mathrm{H}_{52} \mathrm{NO}_{2}[\mathrm{M}+\mathrm{H}]^{+}$506.3998, found 506.4009.

\section{1 .226 -羟基胆甾-3-芐氧基肪(25)的合成}

制备方法与化合物 $\mathbf{4}$ 类似, 以化合物 $\mathbf{2 4}$ 为原料, 得 到白色固体 25, 产率 65\%. m.p. 146 $148{ }^{\circ} \mathrm{C} ;{ }^{1} \mathrm{H} \mathrm{NMR}$ $\left(\mathrm{CDCl}_{3}, 300 \mathrm{MHz}\right) \delta: 0.72\left(\mathrm{~s}, 3 \mathrm{H}, 18-\mathrm{CH}_{3}\right), 0.887(\mathrm{~d}, J=$ $6.6 \mathrm{~Hz}, 3 \mathrm{H}, 26-\mathrm{CH}_{3}$ or $\left.27-\mathrm{CH}_{3}\right), 0.891(\mathrm{~d}, J=6.6 \mathrm{~Hz}, 3 \mathrm{H}$, $26-\mathrm{CH}_{3}$ or $\left.27-\mathrm{CH}_{3}\right), 0.93\left(\mathrm{~d}, J=6.3 \mathrm{~Hz}, 3 \mathrm{H}, 21-\mathrm{CH}_{3}\right), 1.12$ (s, 3H, 19- $\mathrm{CH}_{3}$ ), 2.02 (dt, $\left.J=12.6,3.2 \mathrm{~Hz}, 1 \mathrm{H}, \mathrm{C}^{8}-\mathrm{H}\right), 2.12$ (br d, $J=14.7 \mathrm{~Hz}, 1 \mathrm{H}, \mathrm{C}^{4}-\mathrm{H}$ ), 2.57 (t, $J=13.8 \mathrm{~Hz}, 1 \mathrm{H}$, $\left.\mathrm{C}^{5}-\mathrm{H}\right), 3.25\left(\mathrm{dd}, J=15.3,4.8 \mathrm{~Hz}, 1 \mathrm{H}, \mathrm{C}^{2}-\beta-\mathrm{H}\right), 3.86 \sim 3.80$ $\left(\mathrm{m}, 1 \mathrm{H}, \mathrm{C}^{6}-\mathrm{H}\right), 5.08\left(\mathrm{~s}, 2 \mathrm{H}, \mathrm{OCH}_{2}\right), 7.39 \sim 7.29(\mathrm{~m}, 5 \mathrm{H}$, $\left.\mathrm{C}_{6} \mathrm{H}_{5}\right) ;{ }^{13} \mathrm{C} \mathrm{NMR}\left(\mathrm{CDCl}_{3}, 75 \mathrm{MHz}\right) \delta: 160.9$ (3-C), [127.6, $127.9,127.9,128.3,128.3,138.3]\left(\mathrm{C}_{6} \mathrm{H}_{5}\right), 75.2\left(\mathrm{OCH}_{2}\right)$, 71.1 (6-C), 56.3 (14-C), 56.1 (17-C), 53.9 (9-C), 49.1 (5-C), 42.7 (13-C), 39.9 (10-C), 39.8 (7-C), 39.5 (12-C), 39.0 (24-C), 36.2 (22-C), 36.1 (20-C), 35.8 (8-C), 31.7 (4-C), 30.2 (16-C), 28.2 (25-C), 28.0 (2-C), 24.2 (1-C), 23.8 (15-C), 22.8 (26-C), 22.6 (27-C), 21.5 (23-C),21.1 (11-C), 18.7 (21-C), 14.9 (19-C), 12.1 (18-C); IR (KBr) v: 3370, 2929, 2859, 1638, 1454, 1364, $1033 \mathrm{~cm}^{-1}$; HREIMS calcd for $\mathrm{C}_{34} \mathrm{H}_{54} \mathrm{NO}_{2}[\mathrm{M}+\mathrm{H}]^{+}$508.4155, found 508.4144.

\section{2 体外抗肿瘤活性研究}

使用顺铂(Cisplatin)作为阳性对照, 采用四甲基偶 氮唑盐(MTT)法测试所合成的目标化合物对人胃癌细胞 (SGC-7901)、人肝癌细胞 (Bel-7404) 和鼻咽癌细胞 (CNE-2)的体外生长增殖抑制活性. 所有细胞分别采用 RPMI-1640 培养液(加入 $10 \%$ 牛血清和 $0.1 \mathrm{~g} / \mathrm{L}$ 青霉素
$\mathrm{G}+0.1 \mathrm{~g} / \mathrm{L}$ 硫酸链霉素)在 $37{ }^{\circ} \mathrm{C}$ 于 $5 \% \mathrm{CO}_{2}$ 的潮湿气氛 中培养.

实验步骤如下: 步骤 1: 首先将有机化合物溶解在 DMSO 中, 配制成 $10 \mathrm{mg} / \mathrm{mL}$ 的 DMSO 溶液保存在冰箱; 步骤 2: 将对数生长期的肿瘤细胞分别以约 $(3 \sim 4) \times 10^{4}$ 个 $/ \mathrm{mL}$ 的密度接种于 96 孔板中, 每孔接种 $200 \mu \mathrm{L}$, 置于 $\mathrm{CO}_{2}$ 培养箱中培养 $24 \mathrm{~h}$; 步骤 3: 按预设的浓度梯度(最 高浓度设为 $80 \mu \mathrm{g} / \mathrm{mL}$ ) 加入待测样品, 每一浓度梯度设 3 个平行孔，同时分别采用顺铂作为阳性对照和等量的 DMSO 作为阴性对照; 步骤 4: 在二氧化碳培养箱中于 $37{ }^{\circ} \mathrm{C}$ 培养 $72 \mathrm{~h}$ 后, 每孔加入 $20 \mu \mathrm{L}$ 的 MTT $(5 \mathrm{mg} / \mathrm{mL})$, 然后在二氧化碳培养箱中继续温育 $4 \mathrm{~h}$; 步骤 5: 抽取上 清液, 然后加入 $200 \mu \mathrm{L}$ 的 DMSO, 在振动器上震荡 10 $\min$ 溶解沉淀, 随后用酶标仪在 $490 \mathrm{~nm}$ 波长测定 OD 值. 通过下式求出样品一定浓度下样品对细胞的抑制率:

\section{抑制率 $=[($ 对照 $\mathrm{OD}$ - 空白 $\mathrm{OD})-($ 给药 $\mathrm{OD}-$ \\ 空白 OD) $] /($ 对照 OD一空白 OD) $\times 100 \%$}

然后以抑制率对药物浓度作图, 求出每个样品的 $\mathrm{IC}_{50}$ 值. 如果化合物在最高浓度没有抑制作用，一般测 定 1 次, 其它的每个样品平行测定 3 次, 计算平均值, 偏 差大的需要进一步测定.

\section{References}

[1] Gobbini, M.; Armaroli, S.; Banfi, L.; Benicchio, A.; Carzana, G.; Fedrizzi, G.; Ferrari, P.; Giacalone, G.; Giubileo, M.; Marazzi, G.; Micheletti, R.; Moro, B.; Pozzi, M.; Scotti, P. E.; Torri, M.; Cerri, A. J. Med. Chem. 2008, 51, 4601.

[2] Cushman, M.; He, H.-M.; Katzenellenbogen, J. A.; Varma, R. K.; Hamel, E.; Lin, C. M.; Ram, S.; Sachdeva, Y. P. J. Med. Chem. 1997, 40, 2323.

[3] Huang, Y.-M.; Cui, J.-G.; Zhong, Z.-G.; Gan, C.-F.; Zhang, W.-Y.; Song, H.-C. Bioorg. Med. Chem. Lett. 2011, 21, 3641.

[4] Shi, J.-F.; Xu, F.; Liao Q.-J. J. China Pharm. Univ. 1987, 18, 169 (in Chinese).

(施菊芳, 徐芳, 廖清江, 中国药科大学学报, 1987, 18, 169.)

[5] Khana, S. A.; Asiri, A. M.; Saleem, K. J. Saudi Chem. Soc. 2012, 16,7 .

[6] Fan, L.; Cui, J.-G.; Wei, Y.-L.; Huang, Y.-M. Mod. Agrochem. 2008, 7, 6 (in Chinese). (范否, 崔建国, 韦英亮, 黄燕敏, 现代农药, 2008, 7, 6.)

[7] Jindal, D. P.; Chattopadhaya, R.; Guleria, S.; Gupta, R. Eur. J. Med. Chem. 2003, 38, 1025.

[8] Lee, J.; Zylka, M. J.; Anderson, D. J.; Burdette, J. E.; Woodruff, T. K.; Meade, T. J. J. Am. Chem. Soc. 2005, 127, 13164.

[9] Khan, S. A.; Asiri, A. M.; Saleem, K. J. Saudi Chem. Soc. 2012, 16, 7.

[10] Wu, X.-D.; Liu, D.-Z.; Zhou, X.-Q.; Yang, X.-W.; Zhu, K.-M. Acta Chim. Sinica 2009, 67, 1487 (in Chinese).

(吴学丹, 刘东志, 周雪琴, 杨雄文, 朱孔明, 化学学报, 2009, 67, 1487.)

[11] Cerny, I.; Pouzar, V.; Hill, M.; Havlikova, H.; Hampl, R. Steroids 2006, $71,120$.

[12] Morris, K. D. W.; Amin, J. Mol. Pharmacol. 2004, 66, 56. 
[13] Cui, J.-G.; Huang, L.-L.; Fan, L.; Zhou, A.-M. Steroids 2008, 73, 252.

[14] Cui, J.-G.; Fan, L.; Huang, L.-L.; Liu, H.-L.; Zhou, A.-M. Steroids 2009, 74, 62 .
[15] Cui, J.-G.; Fan, L.; Huang, Y.-M.; Xin, Y.; Zhou, A.-M. Steroids 2009, 74, 989 .

[16] Takatsuto, S.; Ikekewa, S. N. J. Chem. Soc., Perkin Trans. 1 1984, 439.

(Li, L.; Lu, Z.) 